NBER WORKING PAPER SERIES

\title{
A SEARCH COST MODEL OF OBFUSCATION
}

\author{
Glenn Ellison \\ Alexander Wolitzky \\ Working Paper 15237 \\ http://www.nber.org/papers/w15237
NATIONAL BUREAU OF ECONOMIC RESEARCH
1050 Massachusetts Avenue
Cambridge, MA 02138
August 2009

This paper was supported by NSF grant SES-0550897 and the Toulouse Network for Information Technology. The second author also received support through a NSF Graduate Research Fellowship. The views expressed herein are those of the author(s) and do not necessarily reflect the views of the National Bureau of Economic Research.

NBER working papers are circulated for discussion and comment purposes. They have not been peerreviewed or been subject to the review by the NBER Board of Directors that accompanies official NBER publications.

(C) 2009 by Glenn Ellison and Alexander Wolitzky. All rights reserved. Short sections of text, not to exceed two paragraphs, may be quoted without explicit permission provided that full credit, including (C) notice, is given to the source. 
A Search Cost Model of Obfuscation

Glenn Ellison and Alexander Wolitzky

NBER Working Paper No. 15237

August 2009

JEL No. D43,D83,L13

\begin{abstract}
This paper develops search-theoretic models in which it is individually rational for firms to engage in obfuscation. It considers oligopoly competition between firms selling a homogeneous good to a population of rational consumers who incur search costs to learn each firm's price. Search costs are endogenized: obfuscation is equated with unobservable actions that make it more time-consuming to inspect a product and learn its price. We note two mechanisms by which obfuscation can affect consumer beliefs about future search costs: a direct effect that applies when search costs are convex in time spent searching and a signal-jamming effect that applies when an informational link is present. As long as obfuscation is costless for firms, the presence of either of these mechanisms guarantees that obfuscation must occur in equilibrium, unless consumer search costs are already so high that consumers are willing to purchase at the highest equilibrium price in the absence of obfuscation. Changes in consumer search costs are at least partially offset by changes in the equilibrium level of obfuscation, raising doubts about whether reductions in consumer search costs must make markets more competitive. We also examine patterns of obfuscation and show that higher markups are usually associated with more obfuscation.
\end{abstract}

Glenn Ellison

Department of Economics

Massachusetts Institute of Technology

50 Memorial Drive, E52-380A

Cambridge, MA 02142-1347

and NBER

gellison@mit.edu

Alexander Wolitzky

Department of Economics

Massachusetts Institute of Technology

50 Memorial Drive

Cambridge, MA 02142-1347

wolitzky@mit.edu 


\section{Introduction}

Anyone who has shopped for a mattress, tried to compare the full sets of fees charged by multiple banks or mortgage lenders, or gotten quotes from contractors for a home renovation will find it easy to question the universality of the classic economic argument that firms will disclose all relevant information. ${ }^{1}$ Ellison and Ellison (2009) describe practices in which firms intentionally make shopping complicated, difficult, or confusing as "obfuscation" and provide empirical evidence from online shopping. It is easy to think of reasons why it would be collectively rational for firms to practice obfuscation: equilibrium prices are increasing in consumer search costs in many search models, and price discrimination arguments can also be given. ${ }^{2}$ Arguments based on collective rationality, however, bring up a natural critique: why collude on obfuscation rather than just colluding directly on price? In this paper, we discuss a search-based model in which it is individually rational for firms to raise consumer search costs.

Diamond (1971) first formalized the connection between search costs and price levels, noting that even an $\epsilon$ search cost could increase prices from the competitive level to the monopoly level because consumers will have no incentive to search if they expect all firms to charge monopoly prices. Several subsequent papers developed two other important insights: there is a more natural search problem when price dispersion is present, and price dispersion will exist in equilibrium when consumers are differentially informed. ${ }^{3}$ Our model closely follows that of Stahl (1989), who considers a continuum of consumers shopping for a homogenous good offered by $N$ firms. A fraction $\mu$ of the consumers have no search costs and learn all firms' prices. The other $1-\mu$ pay a search cost of $s$ every time they obtain a price quote. Consumers have identical downward sloping demands $D(p)$. Stahl shows that this produces an elegant, tractable model. All consumers with positive search costs search exactly once. Firms choose prices from a nonatomic distribution on an interval $[\underline{p}, \bar{p}]$

\footnotetext{
${ }^{1}$ See Grossman (1981) and Milgrom (1981).

${ }^{2}$ Diamond(1971) and many subsequent papers connect search costs and equilibrium price levels. Ellison (2005) shows that the joint adoption of add-on pricing strategies can increase prices in a competitive price discrimination model.

${ }^{3}$ Classic papers include Butters (1977), Salop and Stiglitz (1977), Varian (1980), Rosenthal (1980), and Burdett and Judd (1983). See Baye and Morgan (2001) for a recent model applied to online markets and Baye, Morgan, and Scholten (2006) for a nice survey including recent developments.
} 
following mixed strategies like those in Varian (1980) and Rosenthal (1980). The model's comparative statics clearly bring out the collective incentive to increase search costs: prices and firm profits increase as the search cost $s$ increases.

Section 2 of our paper introduces our model and derives some preliminary results that are common to the different versions we eventually consider. We model obfuscation in a very simple way: consumers are assumed to have a disutility that depends on the the total time spent shopping, and each firm is allowed to choose the length of time that is required to learn its price. In the bank application, for example, the firm may be choosing the complexity of its fee structure which determines how long it would take a consumer to read through the full list of fees for overdrafts, low balances, ATM use, wire transfers, etc. and estimate what he or she will end up paying each month. ${ }^{4}$ This time is not observable to consumers until after they have visited the firm. A number of the basic features of Stahl's model carry over to our environment: in equilibrium, firms make positive profits and choose prices from a nonatomic distribution with support $[\underline{p}, \bar{p}]$; and consumers search until the expected gain from taking another draw from the price distribution exceeds the expected search costs.

Section 3 analyzes our simplest model of obfuscation. On the firm side obfuscation is assumed to be costless. On the consumer side we assume that consumers have a strictly convex disutility $g(t)$ for the time $t$ they spend shopping. We view this as a small departure from the traditional assumption in a realistic direction: disutility would be convex in a standard time-allocation model with decreasing returns to leisure; and for many results $g^{\prime}(t)$ need only be $\epsilon$ greater than $g^{\prime}(0)$ even in the $t \rightarrow \infty$ limit. Yet, it is a departure that can greatly alter the equilibrium set. Holding obfuscation levels fixed our model is much like Stahl's and the firms' pricing strategies will coincide with those of Stahl's model, with the search cost parameter set equal to the incremental costs of a second search. The possibility of doing slightly more obfuscation than consumers expect, however, can have a dramatic

\footnotetext{
${ }^{4}$ The "price" the consumer is learning can also be interpreted as the time required to learn the product' quality and thereby learn a quality-adjusted price. For example, in the case of mattress shopping the price of each mattress at a store, e.g. "Sealy Posturepedic Ruby", may be readily observable but time will be required to inquire about product attributes and learn which name corresponds to the mattress the consumer had seen at another store and/or to make mental adjustments to account for differences in the attributes of different stores' offerings.
} 
effect on what is possible in equilibrium. Specifically, equilibria in which the upper bound of the price distribution is strictly less than the monopoly price become impossible because a firm can simultaneously make small deviations in two dimensions: increase its price to slightly above $\bar{p}$ and also slightly increase its obfuscation level. Hence, in all equilibria of our model the upper bound of the price distribution is the monopoly price. Such an upper bound on the equilibrium distribution is only possible if equilibrium search costs are above some lower bound. Therefore, there is a lower bound on the level of equilibrium obfuscation. The lower bound can be zero, but can also be substantial. Obfuscation hurts consumers in two ways: consumers incur higher search costs and pay higher prices.

Section 4 considers costly obfuscation. This makes obfuscation levels more deterministic, because in equilibrium each firm must be choosing the minimum level of obfuscation consistent with the equilibrium level of consumer search. It also allows us to discuss crosssectional relationships between prices and obfuscation. For example, in some circumstances we note that firms with the lowest markups will not obfuscate at all, whereas firms with the highest markups can only make sales if they are doing substantial obfuscation. We present results from two different modeling approaches. One is an equilibrium refinements approach which can be motivated by thinking of the cost of obfuscating as lexicographically less important to firms than profits. The other is a more traditional model.

Section 5 considers an alternate mechanism for producing obfuscation. We return to the traditional assumption that consumers have a linear disutility of search effort, dropping the strict convexity assumption used in sections 3 and 4. Instead, we depart from Stahl's model in another direction we find realistic: we assume that there is common uncertainty about how much time is required to learn a firm's price in the absence of obfuscation. A key feature of such models is that consumers' expectations about future search costs increase in the amount of time it takes them to learn the price of the first firm they visit. For example, one could think of this as a model in which consumers are not born knowing how long it takes to get a price quote from a home improvement contractor and in which consumers who spend a long time discussing a project with the first contractor they contact will assume that the process of getting a bid from another contractor will also be time-consuming. A natural consequence of such an effect is that obfuscation can occur for signal-jamming 
reasons. Some predictions of the signal-jamming model are similar to the convex costs model: we provide conditions under which some obfuscation must occur and show that the equilibrium price distributions are a selection from the set of equilibrium distributions of Stahl's model. But the mechanism behind the obfuscation is somewhat different and this leads to some interesting differences in predictions. One is an "excess obfuscation" result noting that obfuscation is almost always above what is necessary to deter search. Another is that the selection among the equilibria of Stahl's model is different and that excess obfuscation can eliminate equilibria with very high prices. Some comparative statics results also differ across the models.

Our paper is related to a number of others. Ellison and Ellison (2009) provide informal descriptive evidence on obfuscation among a group of e-retailers and present empirical evidence that suggests that at least two mechanisms are involved: consumers appear to have a substantially incomplete knowledge of prices, and firms' add-on pricing strategies appear to create an adverse-selection effect that would be expected to increase equilibrium markups. A number of subsequent papers have explored obfuscation mechanisms. ${ }^{5}$ Ellison (2005) discusses add-on pricing in the context of a competitive price discrimination model. It notes that add-on pricing is not individually rational in the base model, but could be made individually rational by adding a subpopulation of irrational consumers who were exploited by the add-on strategy. Gabaix and Laibson (2006) work out an explicit model along these lines.

Spiegler (2006) provides an alternate boundedly-rational approach. In his model, consumers are only capable on evaluating products on one of many dimensions. Firms "obfuscate" by randomizing and making the product more attractive on some dimensions (e.g. making fees lower if some contingency arises) and less attractive on others. He notes that an increase in the competitiveness of the market (more firms) leads to an increase in obfuscation but no change in average prices. This is a somewhat similar in spirit to our finding that decreases in exogenous search costs don't change average prices because they are fully offset by a change in obfuscation. But the meaning of obfuscation and the mechanisms are, of course, completely different. His model and ours may differ in other predictions.

\footnotetext{
${ }^{5}$ Ellison (2006) includes a survey of some of this literature.
} 
Eliaz and Spiegler (2008) address some related topics, e.g. whether firms with higher prices do more or less to inform consumers, in another elegant model with boundedly rational consumers. Their model, however, is more similar in spirit to the traditional information revelation literature than to our paper in that informing consumers is the costly action.

Carlin (2008) and Wilson (2008) are most closely related to our paper. Each also models obfuscation as a strategic decision by firms that increases search costs in a model with optimal consumer search. Carlin's model differs from ours both in the focus and in the type of search model it uses. The search model is an all-or-nothing model along the lines of Salop and Stiglitz (1977) and Varian (1980). ${ }^{6}$ More importantly, Carlin's (2008) focus is primarily on how obfuscation affects market prices, whereas some of our main motivations are to explore why it is individually (as opposed to collectively) rational to obfuscate and how obfuscation varies in the cross-section. Carlin (2008) does make obfuscation individually rational and not just collectively rational, but this is done in a fairly straightforward manner so the paper can focus on other things: consumers observe a summary statistic (like the average obfuscation level) before deciding whether to conduct an all-or-nothing search and do not observe any individual firm's obfuscation level, so an increase in obfuscation by any one firm leads to exactly the same outcome as would a smaller coordinated increase by all firms.

Wilson (2008) does focus on the question of why obfuscation is individually rational and develops a very nice argument (which is also very different from ours). The primary difference between Wilson's model and ours is that Wilson assumes that the firm-specific search costs are observable to consumers when they choose which stores to visit. One's first thought might be that this will make obfuscation impossible, because consumers will always choose to visit firms with the lowest search costs first. Wilson's clever observation is that while it is true that many or all consumers will visit the low-search-cost firm first, this does not necessarily render obfuscation unappealing. Obfuscation can provide strategiccommitment benefits: by making itself less attractive to the consumers with positive search costs, the obfuscating firm induces its rival to focus more on these consumers and raise prices, which can benefit both firms. Our paper differs from his in the assumptions on

\footnotetext{
${ }^{6}$ Baye, Morgan, and Scholten (2006) refer to these as clearinghouse models.
} 
observability, in the mechanisms that drive obfuscation, and in the details of many results. For example, in his paper obfuscating firms tend to charge lower prices, whereas obfuscation is associated with charging high prices in our model.

\section{$2 \quad$ Model and Preliminary Results}

In this section we present our model and derive some basic results. Our model is similar to that of Stahl (1989) with two additions: search costs are allowed to be a nonlinear function of the number of searches carried out; and the per-search cost is an endogenous choice of the firms. The results in this section show that some standard results carry over: consumer search strategies can be characterized using standard cutoff rules, firms earn positive profits in a dispersed price equilibrium, and equilibrium price distributions are atomless.

\subsection{Model}

We consider a unit mass of consumers of two types: proportion $\mu$ are "costless searchers" who automatically learn all firms' prices and proportion $1-\mu$ are "costly searchers" who must incur search cost $g(t)$ to spend a total time $t$ searching. We assume that ascertaining firm $i$ 's price requires time $\tau+t_{i}$, where $\tau>0$ is exogenous and $t_{i}$ is the obfuscation level chosen by firm $i$. For example, $\tau$ might be the amount of time it takes to drive to a store or to access a website, whereas $t_{i}$ might be the amount of time it takes to discover the relevant price after the store is reached or the website is opened. ${ }^{7}$ Therefore, a costly searcher would incur cost $g\left(\tau+t_{1}\right)$ to learn the price of a firm that chooses obfuscation level $t_{1}$ if this is her first search and would incur total cost $g\left(2 \tau+t_{1}+t_{2}\right)$ if she chose to continue her search and also learn the price of a second firm that chooses obfuscation level $t_{2}$. We assume that $g(\cdot)$ is twice continuously differentiable, strictly increasing, and weakly convex with $g(0)=0$. We assume that consumers cannot observe a firm's obfuscation level before they visit it and learn its price, but do have rational expectations about the distribution of obfuscation

\footnotetext{
${ }^{7}$ We assume throughout that consumers can choose to go back to a previously visited firm at zero cost. This would fit the example of Internet search if consumers leave open a browser window containing the best price they have found. The driving example does not fit this property well if consumers must drive back to a previously visited store to purchase from it, but fits it better if consumers can call the store back on the phone to order a previously researched product.
} 
levels. $^{8}$

As in Stahl (1989), we assume that consumers have downward-sloping demand functions $D(p)$ that satisfy $\int_{0}^{z} D(x) d x<\infty$ for all $z \geq 0$ and let $R(p) \equiv p D(p)$, the revenue a firm obtains from selling to consumer with demand $D(p)$ at price $p$. We assume that $R(p)$ is continuously differentiable with unique maximum $p^{m}$ and that $R^{\prime}(p)>0$ if $p<p^{m}$. Each firm $i$ out of $N \geq 2$ firms chooses price $p_{i}$ and obfuscation level $t_{i}$. Firms produce at zero marginal cost. Firm $i$ incurs a fixed obfuscation cost of $c\left(t_{i}\right)$ when it chooses obfuscation level $t_{i}$; we assume that the obfuscation cost function is differentiable with $c(0)=0$ and $c^{\prime}(t) \geq 0$ for all $t$. In some sections we will focus on the case of costless obfuscation, $c(t)=0$ for all $t$, which allows for the simplest results.

The game proceeds as follows. First, firms simultaneously and noncooperatively choose obfuscation levels and prices. Then, costless searchers automatically learn all firms' prices and can buy from any firm, and costly searchers search strategically: they draw a new, randomly selected firm with each search and may stop searching and buy from any firm they have visited at any point. We say that costly searchers "enter" if they choose to search at least once. We will look for symmetric Nash Equilibria of this game ${ }^{9}$; henceforth by "NE" we mean symmetric Nash Equilibria. ${ }^{10}$

\subsection{Search strategies}

In this section we show that standard results on optimal search strategies carry over to our model. To state this formally, note first that every symmetric strategy profile induces a price distribution $F(p)$. If the price distribution is given by $F(p)$ and a consumer has already spent total time $t_{0}$ searching and has observed price $p_{0}$ but no lower prices, then the consumer's expected cost to searching again is $\mathbb{E}_{t}\left[g\left(t_{0}+\tau+t\right)-g\left(t_{0}\right)\right]$, whereas her expected benefit from searching again and then buying from the lowest-price firm she has

\footnotetext{
${ }^{8}$ In some of our models one could alternately regard consumers as learning the $t_{i}$ after incurring only the $\operatorname{cost} \tau$, with the option to discontinue search at this point, but such interpretations will require some strong refinement on beliefs to prevent bootstrapped equilibria in which consumers assume that a firm offers a very unattractive price if its obfuscation level is not exactly equal to some value.

${ }^{9}$ Note that as usual the assumption that costless searchers automatically learn all firms' prices rather than searching sequentially with zero search cost is substantive. If we assumed the latter, all firms' setting price equal to $p^{m}$ would be an equilibrium, for example.

${ }^{10}$ Our assumption that consumers search randomly among firms would be unappealing if firms did not follow symmetric strategies.
} 
observed is

$$
\begin{aligned}
V\left(p_{0}\right) & \equiv \int_{\underline{p}}^{p_{0}}\left(C S(x)-C S\left(p_{0}\right)\right) f(x) d x \\
& =\int_{\underline{p}}^{p_{0}}\left(\int_{x}^{\infty} D(p) d p-\int_{p_{0}}^{\infty} D(p) d p\right) f(x) d x \\
& =\int_{\underline{p}}^{p_{0}}\left(\int_{x}^{p_{0}} D(p) d p\right) f(x) d x \\
& =\int_{\underline{p}}^{p_{0}}\left(\int_{\underline{p}}^{p} f(x) d x\right) D(p) d p \\
& =\int_{\underline{p}}^{p_{0}} D(p) F(p) d p
\end{aligned}
$$

where $\underline{p}$ is the infimum of the support of $F(p)$, and $C S(p)$ is the consumer surplus at price $p$.

We begin by showing that optimal consumer search is given by continuing search if $\mathbb{E}_{t}\left[g\left(t_{0}+\tau+t\right)-g\left(t_{0}\right)\right]<V\left(p_{0}\right)$ and by stopping search if $\mathbb{E}_{t}\left[g\left(t_{0}+\tau+t\right)-g\left(t_{0}\right)\right]>$ $V\left(p_{0}\right) \cdot{ }^{11}$

Proposition 1 In any NE, a costly searcher stops searching if $V\left(p_{0}\right)<\mathbb{E}_{t}\left[g\left(t_{0}+\tau+t\right)-g\left(t_{0}\right)\right]$ or if all $N$ firms have been visited and continues searching if $V\left(p_{0}\right)>\mathbb{E}_{t}\left[g\left(t_{0}+\tau+t\right)-g\left(t_{0}\right)\right]$.

We present a formal proof in the appendix. It proceeds by induction on the number of stores remaining using a two case argument: if the incremental cost of the next search is less than $V\left(p_{0}\right)$, then searching must be optimal because searching exactly once is better than not searching; and if the incremental cost is greater than $V\left(p_{0}\right)$, then not searching must be optimal because continuing to search is less appealing than it would be if incremental search costs were constant, and standard results imply that continuing is not optimal in that case.

\subsection{Price equilibrium}

In this section we recall some standard results for the case where $t$ is a parameter rather than a choice variable and show that properties of these equilibria carry over to our model.

\footnotetext{
${ }^{11}$ This is not a direct corollary of the classic results since here the expected search costs faced by consumers depends on the entire history of the obfuscation levels they have encountered.
} 
Before doing so, we should note that our model sometimes has equilibria in which the costly searchers are inactive. If exogenous or endogenous search costs are sufficiently high, then costly searchers will not get even a single price quote. We will mostly ignore these equilibria and use the phrase "nontrivial NE" to mean a NE in which the costly searchers do get at least one price quote. ${ }^{12}$

Proposition 2 (Stahl 1989) Suppose that every firm's level of obfuscation is fixed exogenously at $t$. Then the price distribution for any nontrivial NE takes one of two possible forms:

1. If there exists an $r \in\left(0, p^{m}\right)$ for which

$$
\int_{\underline{p}}^{r} D(p)\left(1-\left[\left(\frac{1-\mu}{N \mu}\right)\left(\frac{R(\bar{p})}{R(p)}-1\right)\right]^{\frac{1}{N-1}}\right) d p=g(2(\tau+t))-g(\tau+t),
$$

then the equilibrium price distribution is $F(p)=1-\left[\left(\frac{1-\mu}{N \mu}\right)\left(\frac{R(\bar{p})}{R(p)}-1\right)\right]^{\frac{1}{N-1}}$, with $\bar{p}=r$, and $R(\underline{p})=\left[\frac{1-\mu}{1+(N-1) \mu}\right] R(\bar{p})$.

2. If there does not exist such a value of $r$, then the equilibrium price distribution is

$$
F(p)=1-\left[\left(\frac{1-\mu}{N \mu}\right)\left(\frac{R(\bar{p})}{R(p)}-1\right)\right]^{\frac{1}{N-1}}, \bar{p}=p^{m} \text {, and } R(\underline{p})=\left[\frac{1-\mu}{1+(N-1) \mu}\right] R(\bar{p}) .
$$

Proof. By Proposition 1, costly searchers search for a second time after observing price $p_{0}$ if $V\left(p_{0}\right)>g(2(\tau+t))-g(\tau+t)$ and do not search for a second time if $V\left(p_{0}\right)<g(2(\tau+t))-g(\tau+$ $t$ ). The result then follows immediately from Stahl's analysis for $s=g(2(\tau+t))-g(\tau+t)$.

Note that Proposition 2 can be thought of as showing that two slightly different types of mixed equilibria arise. The first type arises when search costs are small. In these equilibria, the constraint that consumers must be willing to buy from a firm offering price $\bar{p}$ rather than searching again is binding and pins down the upper bound of the support of the price distribution. The upper bound of the support and the distribution of prices vary with the search cost in these equilibria. The second type arises when search costs are larger. In these

\footnotetext{
${ }^{12}$ In a trivial $\mathrm{NE}$ the fact that only costless searchers are in the market implies that firms are Bertrand competitors, so firms must price at cost (or at least two firms must do so without the symmetry restriction). Obfuscation levels would need to be high enough so that costly searchers nonetheless do not want to enter.
} 
equilibria, consumers strictly prefer buying from the first firm they visit to getting another price quote. The upper bound of the price distribution is always the monopoly price. And the price distribution is also independent of the search cost over the range of search costs for which this case applies.

When $t$ is exogenously fixed, it is well-known that every NE price distribution is atomless, that every firm makes positive profits in any NE, and that in any NE every costly searcher buys from the first firm she visits. The former two results continue to hold generally when $t$ is a choice variable, while the last result requires the additional assumption that obfuscation is costless for firms, which we impose in Section 3.

Lemma 1 Every firm makes positive profits in any nontrivial NE.

Proof. Setting a strictly negative price is strictly dominated, so in any nontrivial NE all prices are weakly positive. Therefore, if the first price a costly searcher observes is $p_{0}$, then in NE her benefit from searching again if every firm sets $t_{i}=0$ is $\int_{0}^{p_{0}} D(x) F(x) d x-$ $(g(2 \tau)-g(\tau)) \leq \int_{0}^{p_{0}} D(x) d x-(g(2 \tau)-g(\tau))$, which is negative for $p_{0}$ sufficiently close to 0 . By convexity of $g$, her benefit from searching again is no greater than this if any firm sets positive obfuscation. Therefore, in NE any firm can guarantee itself positive profits by choosing such a sufficiently small but strictly positive $p_{0}$, so every firm must make positive profits in any NE.

Lemma 2 If $F(p)$ is a nontrivial NE price distribution, then it is atomless.

Proof. By Lemma 1, no firm sets $p=0$ in any NE in which costly searchers enter. So if $F(p)$ has an atom, it must have an atom at some $p>0$. But then pricing slightly below this atom yields strictly higher profits than pricing at the atom, as it yields a discrete gain in profits from the costless searchers and an arbitrarily small loss in profits from the costly searchers.

Lemma 3 If $c(t)=0$ for all $t$, then on the equilibrium path of any NE every costly searcher searches at most once.

Proof. Let $F(p)$ be a nontrivial NE price distribution for a model with costless obfuscation. Let $\bar{p}$ be the maximum of the support of $F(p)$. Consider a firm that sets price equal to $\bar{p}$. If 
this firm does not sell to any of the costly searchers that visit it first, then with probability 1 it will not sell to any consumers as, by Lemma 2, every other firm has a lower price with probability 1 and consumers buy from the lowest-priced firm they visit. This would contradict Lemma 1, so a firm that sets price equal to $\bar{p}$ must sell to some costly searchers that visit it first. Furthermore, if consumers mix between buying and not buying from a firm with price equal to $\bar{p}$, then by lowering prices by an arbitrarily small amount the firm could sell to these consumers with probability 1, by Proposition 1, strictly increasing profits. So if $F(p)$ is a NE price distribution then every costly searcher who visits a firm with price equal to $\bar{p}$ first buys immediately.

Since those costly searchers who first visit a highest-priced firm buy from it, any lowerpriced firm could sell to those costly searchers who visit it first by setting the same obfuscation level as the highest-priced firm. And raising one's obfuscation level only increases the number of consumers one sells to, by our characterization of optimal consumer search, so if a lower-priced firm did not sell to those costly searchers who visited it first it could strictly increase profits by raising its obfuscation level to that of the highest-priced firm.

Note that for Lemma 3 we added an assumption that obfuscation is costless. We believe that this is necessary given that we have made few assumptions on the $g(t)$ function. To see why, consider, for example, a $g(t)$ function which is zero up some point and then follows a strictly convex function with unbounded slope. In this case, firms have the option of deterring future searches by customers who visit them first, but may need to incur a positive obfuscation cost to do so. In such a model it seems plausible that there could be equilibria in which firms setting low prices choose $t=0$ and accept that all customers who visit them first will obtain a second quote, while firms with prices near the upper bound avoid getting zero demand by choosing an obfuscation level sufficient to ensure that they sell to all consumers who visit them first. 


\section{An Obfuscation Model: Costless obfuscation and convex disutility of search}

In this section we analyze our model under the assumption that obfuscation is costless and consumer disutility for shopping, $g(t)$, is strictly convex. The convex search costs assumption is a departure from much of the previous literature, but we think it is a departure that is worth exploring for two reasons. First, we think it is descriptively accurate: search costs would be convex in a textbook consumer-theory model of time allocation if there are decreasing returns to leisure. Second, it turns out that even small departures from more traditional assumptions in this directions can have dramatic effects on equilibrium outcomes.

The first subsection below discusses the impact that of the convex-search costs assumption on the possible distributions of equilibrium prices. We show that some of the equilibrium price distributions of Stahl's model remain equilibria and some do not. The second subsection discusses equilibrium obfuscation levels. The costlessness of obfuscation results in a degree of indeterminacy, but the result on price distributions implies that there is a lower bound on the amount of obfuscation that can occur in equilibrium. The results only require a slight departure from the more traditional linear search cost assumption in that they will apply even if $g^{\prime}(t)$ is uniformly bounded above by $g^{\prime}(0)+\delta$ for a small positive $\delta$.

\subsection{Equilibrium price distributions}

We may now state our first main result characterizing price distributions with endogenous search costs: if costly searchers search, $F(p)$ is a NE price distribution of the model with endogenous obfuscation if and only if it is a NE price distribution of Stahl's model with search costs fixed at some level and has the upper bound of its support equal to $p^{m}$. In other words, NE price distributions of the second possible form in the characterization of Proposition 2 remain as possible equilibria, whereas equilibrium price distributions of the first form (and all other distributions) cannot arise in our endogenous obfuscation model. 
Proposition $3 F(p)$ is a price distribution for a nontrivial NE only if

$$
F(p)=1-\left[\left(\frac{1-\mu}{N \mu}\right)\left(\frac{R\left(p^{m}\right)}{R(p)}-1\right)\right]^{\frac{1}{N-1}}
$$

for all $p \in\left[\underline{p}, p^{m}\right]$, where $\underline{p}$ is given by $R(\underline{p})=\left[\frac{1-\mu}{1+(N-1) \mu}\right] R\left(p^{m}\right)$. Such a price distribution is a nontrivial NE price distribution if and only if $g(\tau) \leq V(\infty)$.

Proof. We first show that any such $F(p)$ is a NE price distribution if $g(\tau) \leq V(\infty)$. Consider the following equations:

$$
\begin{gathered}
g(2(\tau+t))-g(\tau+t) \geq \int_{\underline{p}}^{p^{m}} D(p) F(p) d p \\
g(\tau+t) \leq \int_{\underline{p}}^{\infty} D(p) F(p) d p
\end{gathered}
$$

Since $g(\cdot)$ is strictly increasing and convex with $g(0)=0$, we have $g(2(\tau+t))-g(\tau+t) \geq$ $g(\tau+t)$ and $\lim _{t \rightarrow \infty} g(2(\tau+t))-g(\tau+t)=\infty$. Together with $g(\tau) \leq V(\infty)$ and $g$ continuous, this implies that there exist $t \in \mathbb{R}^{+}$that satisfy both (2) and (3). Suppose that each firm chooses obfuscation level equal to such a $t$. By equation (3), all consumers will search once; by equation (2) and our characterization of optimal consumer search, no consumer will search more than once. Now a firm that chooses price $p^{m}$ obtains profit $\frac{1-\mu}{N} R\left(p^{m}\right)$, while a firm that chooses price $p<p^{m}$ obtains profit $\left[\mu(1-F(p)]^{N-1}+\frac{1-\mu}{N}\right] R(p)$. It is easy to check that these are equal for all $p \in\left[\underline{p}, p^{m}\right]$ when $F(p)$ is given by equation (1), while profits associated with any $p$ outside this interval are strictly smaller. So $F(p)$ is a NE price distribution supported by any $t$ satisfying equations (2) and (3).

Now suppose that costly searchers enter and that $F(p)$ is not given by equation (1). We consider two cases. First, suppose that $\bar{p} \neq p^{m}$ is such that $F(p)=1-\left[\left(\frac{1-\mu}{N \mu}\right)\left(\frac{R(\bar{p})}{R(p)}-1\right)\right]^{\frac{1}{N-1}}$ for all $p$ in the support of $F$. If $\bar{p}>p^{m}$, a firm could deviate to $p^{m}$ and make strictly higher profits from both costless and costly searchers. Suppose $\bar{p}<p^{m}$. By Lemma 3 , if a firm plays $\left(t_{i}, \bar{p}\right)$ in NE then $\mathbb{E}_{t}\left[g\left(2 \tau+t_{i}+t\right)-g\left(\tau+t_{i}\right)\right] \geq \int_{\underline{p}}^{\bar{p}} D(p) F(p) d p$. So, by strict convexity of $g$, there exist $\varepsilon, \varepsilon^{\prime}$ such that $\mathbb{E}_{t}\left[g\left(2 \tau+t_{i}+\varepsilon+t\right)-g\left(\tau+t_{i}+\varepsilon\right)\right]>$ $\int_{\underline{p}}^{\bar{p}+\varepsilon^{\prime}} D(p) F(p) d p$, so if such a firm deviated to playing $\left(t_{i}+\varepsilon, \bar{p}+\varepsilon^{\prime}\right)$ then a consumer will prefer to buy at price $\bar{p}+\varepsilon^{\prime}$ rather than searching again when the firm's obfuscation level 
is $t_{i}+\varepsilon$. This deviation gives the firm strictly higher profits from the costly searchers and makes no difference to its profits from the costless searchers, since at price $\bar{p}$ it had zero probability of selling to these consumers (by Lemma 2) and still has zero probability of selling to them at price $\bar{p}+\varepsilon$.

Suppose that there exists $p$ in the support of $F$ such that $F(p) \neq 1-\left[\left(\frac{1-\mu}{N \mu}\right)\left(\frac{R(\bar{p})}{R(p)}-1\right)\right]^{\frac{1}{N-1}}$. Then it is easy to check that profits at $p$ do not equal profits at $\bar{p}$, contradicting that $p$ and $\bar{p}$ are both in the support of $F$.

Finally, if $g(\tau)>V(\infty)$, then costly searchers will not search when the price distribution is $F(p)$, so there is no nontrivial NE with price distribution $F(p)$.

Proposition 3 shows that the costless obfuscation model looks much like Stahl's model, with the important difference that equilibria with $\bar{p}<p^{m}$ are ruled out. In particular, any symmetric equilibrium of the costless obfuscation model has the same price distribution as Stahl's model with $\bar{p}=p^{m}$, which is also the same price distribution as in Varian (1980) and Rosenthal (1980).

Note that Proposition 3 makes no assumptions on the consumer search cost function $g$ other than that it is strictly convex. Our intuition for why making $g$ even slightly convex can have a large effect on the equilibrium set follows very much along the lines of the proof: we should think about the possibility that firms can simultaneously deviate to a price slightly above the upper bound of the equilibrium price distribution and an obfuscation level that is slightly higher than consumers expect. In Stahl's model, firms are indifferent between all prices in the interval $[p, \bar{p}]$ because price distributions adjust so that there is a tradeoff between the number of consumers served and the profit per consumer. Equilibria of Stahl's model must also be such that firms do not gain from deviating to a price above $\bar{p}$. This happens because (in equilibria of the first type) this would lead consumers to get another price quote or (in equilibria of the second type) this would be counterproductive because $\bar{p}=p^{m}$. In our model, a firm can raise a consumer's incremental search costs from another search by at least some small amount by increasing its obfuscation level. This would allow the firm to make sales at some price $\bar{p}+\epsilon$. Hence, any equilibrium of Stahl's model with $\bar{p}<p^{m}$ does not survive. 


\subsection{Equilibrium obfuscation levels}

We now consider equilibrium obfuscation levels and note that obfuscation can have substantial effects on search costs and consumer surplus. The fact that all equilibria must have $\bar{p}=p^{m}$ puts a lower bound on equilibrium search costs - consumers must not be willing to conduct a second search when they have found price $p^{m}$ and know that prices are drawn from the distribution given in Proposition 3. If the exogenous component $\tau$ of the search costs is not too large, this implies that firms must obfuscate in equilibrium.

Corollary 1 If $g(2 \tau)-g(\tau)<\int_{\underline{p}}^{p^{m}} D(p) F(p) d p$, where $F(p)$ is given by equation (1), then in any nontrivial $N E$ some firms set $t>0$.

Proof. By Proposition 3, firms are willing to set price equal to $p^{m}$ in any NE in which costly searchers enter. If $g(2 \tau)-g(\tau)<\int_{\underline{p}}^{p^{m}} D(p) F(p) d p$ and $t_{i}=0$ for all $i$, then a costly searcher who first observes a price sufficiently close to $p^{m}$ will search again, contradicting Lemma 3.

We next note the basic welfare consequences of obfuscation. Consumers suffer both directly from the effect of obfuscation on search costs and indirectly because obfuscation leads to higher prices. Firm benefit from the higher prices.

Corollary 2 Suppose $g(2 \tau)-g(\tau)<\int_{\underline{p}}^{p^{m}} D(p) F(p) d p$, where $F(p)$ is given by equation (1). Compared to the model in which obfuscation is impossible ( $t=0$ identically for all firms), the model in which obfuscation is possible leads to higher prices in sense of firstorder stochastic dominance, higher profits for all firms, and lower utility for all consumers in every nontrivial $N E$.

Proof. In the model where $t=0$, the possible nontrivial NE price distributions are given by Proposition 1. If obfuscation is possible, the nontrivial NE price distribution is given by Proposition 3. Since the formula for $F(p)$ is the same in both cases and is decreasing in $\bar{p}$ for all $p$, prices are higher in the sense of first-order stochastic dominance in the latter NE. This and the fact that obfuscation reduces consumer welfare directly imply that in the latter NE firms earn higher profits and consumer welfare is lower. 
One striking fact about Proposition 3 and Corollaries 1 and 2 is that, as long as $g(\tau) \leq$ $V(\infty)$, any reduction in the exogenous fixed component of consumer search costs has no effect whatsoever on the equilibrium distribution of prices and profits - any reduction in $\tau$ that would lead to lower prices must be offset by changes in the equilibrium level of obfuscation. Hence, our model provides a formalization of the observation in Ellison and Ellison (2009) that improvements in search technology need not make search more efficient. Their empirical findings are consistent with the idea that the reduction in search costs online have led to greater equilibrium obfuscation, although probably not with the extreme finding of this section that the response can be so large as to keep the price distribution unchanged. ${ }^{13}$

\subsection{Comparative statics with price-independent obfuscation}

The assumption that obfuscation is costless leads to a degree of indeterminacy in obfuscation levels: obfuscation can vary within a range as long as it is not so low as to induce consumers to search for a second time after seeing price $p^{m}$, nor so high as to deter consumer entry; and obfuscation can also covary with prices in many ways. Here, we provide some comparative statics results by restricting attention to equilibria in which obfuscation levels are independent of the realization of the mixture over prices.

All the comparative statics results in this paper (Propositions 4, 5, 6, 16, 17, 18 and 19) depend on the fact that, if obfuscation is costless, costly searchers must enter and then buy from the first firm they visit in any nontrivial NE. We call these requirements the "entry" and "stopping" conditions; in the current model, when all firms set obfuscation level $t$, these conditions are equations (2) and (3), respectively. The entry condition requires that total search costs be sufficiently low, while the stopping condition requires that total search costs be sufficiently high. Note that, as long as obfuscation is not too concentrated in firms that charge the highest prices, the condition that a consumer who first visits a firm charging $\bar{p}$ does not want to search again is sufficient for the stopping condition. In the current model, both the entry and stopping conditions can always be satisfied simultaneously in equilibria with $\bar{p}=p^{m}$ as long as the entry condition is satisfied when all firms set zero obfuscation

\footnotetext{
${ }^{13}$ The markups they report are lower than one would find in traditional retail.
} 
(i.e. $g(\tau) \leq V(\infty)$ ), because a consumer's benefit from a second search is always lower than the benefit from a first search, while the cost of a second search is always higher than the cost from a first search. We will see that this is no longer true in the signal-jamming model of Section 5 .

Also, since our models may admit multiple equilibria, all comparative statics are on sets of equilibria with respect to the strong set order. Recall that a (one-dimensional) set $X$ is higher than $Y$ in the strong set order if, given elements $x$ in $X$ and $y$ in $Y$, the maximum of $x$ and $y$ is in $X$ while the minimum of $x$ and $y$ is in $Y$.

Our first result identifies a sense in which obfuscation levels must rise when the exogenous component of search costs falls.

Proposition 4 The set of obfuscation values $t^{u}$ (for " $u$ "niform) played in any nontrivial NE in which firms do not mix over obfuscation levels is decreasing in $\tau$, the fixed component of consumer search costs, in the strong set order.

Proof. As in the proof of Proposition 3, the lower bound on $t^{u}$ is given by $g\left(2\left(\tau+t^{u}\right)\right)-g(\tau+$ $\left.t^{u}\right)=\int_{\underline{p}}^{p^{m}} D(p) F(p) d p$ and the upper bound on $t^{u}$ is given by $g\left(\tau+t^{u}\right)=\int_{\underline{p}}^{\infty} D(p) F(p) d p$ so an increase in $\tau$ causes both of these bounds to decrease.

Proposition 4 shows that changes in equilibrium obfuscation offset changes in the exogenous component of search costs. This follows because high exogenous search costs rule out equilibria with high obfuscation, by the entry condition, and eliminate the need for high obfuscation, by the stopping condition. That is, costly searchers will not be willing to obtain a price quote if they face both high exogenous search costs and high obfuscation, and firms have no need to set high obfuscation when consumers are already deterred from comparison-shopping by high exogenous search costs. This effect, however, is weak enough that an increase in $\tau$ must nonetheless lead to a decrease in the set of nontrivial NE values of consumer welfare. The intuition is that prices are fixed by Proposition 3 and any nontrivial NE value of consumer welfare given $\tau^{\prime} \geq \tau$ can be reproduced by uniformly increasing obfuscation by $\tau^{\prime}-\tau$. Note that Proposition 5 does not restrict attention to equilibria with price-independent obfuscation.

Proposition 5 The set of nontrivial NE values of the costly searchers' welfare is decreasing 
in $\tau$ in the strong set order.

Proof. Note that consumer welfare $u$ is given by $u \equiv V(\infty)-\mathbb{E}[g(t+\tau)]$. Suppose that $\tau^{\prime} \geq \tau$ and $u^{\prime} \geq u$, where $u$ is a nontrivial NE value of consumer welfare with fixed search $\operatorname{cost} \tau$ and $u^{\prime}$ is a nontrivial NE value of consumer welfare with fixed search cost $\tau^{\prime}$. We must show that $u^{\prime}$ is a nontrivial NE value with search $\operatorname{cost} \tau$ and that $u$ is a nontrivial NE value with search $\operatorname{cost} \tau^{\prime}$.

First, suppose that $u^{\prime}$ is the value of consumer welfare for a nontrivial NE with price distribution $F(p)$ and obfuscation strategies given as a function of price $t^{\prime}(p)$. Note that $t^{\prime}(p)$ can be a probability distribution over obfuscation levels, if firms mix over obfuscation levels given their prices. Consider the profile where firms price according to $F(p)$ and use obfuscation strategies $t(p)=t^{\prime}(p)+\tau^{\prime}-\tau \geq t^{\prime}(p) \geq 0$, where if $t^{\prime}(p)$ is a probability distribution over obfuscation levels this is interpreted as shifting this distribution up by $\tau^{\prime}-\tau$. It is clear that this profile is a nontrivial NE when fixed search costs are given by $\tau$, because at every history a consumer's expected future total search cost when the fixed component is given by $\tau$ and the variable component is given by $t(p)$ is the same as when the fixed component is given by $\tau^{\prime}$ and the variable component is given by $t^{\prime}(p)$. And consumer welfare in this NE is $u^{\prime}$.

Next, suppose again that $u, u^{\prime}$ and $t^{\prime}(p)$ are as above. To show that $u$ is also a nontrivial $\mathrm{NE}$ value when the exogenous search cost is equal to $\tau^{\prime}$, we suppose that firms draw prices from $F(p)$ and obfuscate according to $t(p)=t^{\prime}(p)+\delta$, where $\delta$ is such that $\mathbb{E}\left[g\left(t^{\prime}(p)+\tau+\right.\right.$ $\left.\delta)-g\left(t^{\prime}(p)+\tau\right)\right]=u^{\prime}-u$. Note first that $t(p) \geq t^{\prime}(p)$ implies that every costly searcher searches at most once, because compared to the original NE, search costs have increased while search benefits remain constant. Second, the fact that the equilibrium utility of each type of consumer is identical to the utility that the same consumer gets in the nontrivial NE with utility $u$ in the game with exogenous search costs $\tau$ implies that costly searchers are willing to enter. Hence, this profile is a nontrivial NE with payoff $u$ in the game with exogenous search costs $\tau^{\prime}$.

In addition one can use the comparative statics presented in Stahl (1989) to derive a number of other comparative statics results. For example, we can show that the lower and 
upper bounds on the NE obfuscation level are both increasing in the proportion of costless searchers. The reason again follows from considering the entry and stopping conditions. When there are more costless searchers, Stahl shows that NE prices are lower in the sense of first-order stochastic dominance. Therefore, more obfuscation is needed to prevent costly searchers who first observe price $p^{m}$ from searching again, so the lower bound on equilibrium obfuscation increases by the stopping condition. Similarly, lower prices imply that costly searchers would be willing to enter despite higher obfuscation, so the upper bound on equilibrium obfuscation increases by the entry condition.

Proposition 6 The set of obfuscation levels $t^{u}$ played in any nontrivial NE where firms do not mix over obfuscation levels is increasing in $\mu$, the proportion of costless searchers, in the strong set order.

Proof. By Proposition 3, $F(p)=1-\left[\left(\frac{1-\mu}{N \mu}\right)\left(\frac{R\left(p^{m}\right)}{R(p)}-1\right)\right]^{\frac{1}{N-1}}$ and $R(\underline{p})=\left(\frac{1-\mu}{1+(N-1) \mu}\right) R(\bar{p})$ in any pure-strategy nontrivial NE, so $F(p)$ is increasing in $\mu$ for all $p$ and $\underline{p}$ is decreasing

in $\mu$. The lower bound on $t^{u}$ is given by $g\left(2\left(\tau+t^{u}\right)\right)-g\left(t^{u}\right)=\int_{\underline{p}}^{p^{m}} D(p) F(p) d p$, so it is increasing in $F(p)$ and therefore increasing in $\mu$. Similarly, the upper bound on $t^{u}$ is given by $g\left(\tau+t^{u}\right)=\int_{\underline{p}}^{\infty} D(p) F(p) d p$, so it is increasing in $F(p)$ and therefore in $\mu$ as well.

One can easily show that the set of values of consumer welfare is increasing in $\mu$. The connection between Proposition 6 and this fact is the same as that between Propositions 4 and 5: raising $\mu$ (or decreasing $\tau$ ) leads to higher obfuscation only because costly searchers benefit more from entering the market when $\mu$ is high (or $\tau$ is low) and are thus willing to tolerate more obfuscation, so obfuscation cannot be so much higher that costly searchers benefit less on net from entering.

\section{Costly Obfuscation}

In some cases, practices that frustrate consumers are simply failures by firms to provide information that they could provide at no (or very little) cost. For example, the computer memory retailers studied in Ellison and Ellison (2009) could easily add pages to their sites explaining what "Cas Latency" means and how important it is or is not for consumers using various applications. In other examples, however, it seems likely that firms incur real 
costs when they engage in obfuscation: mattress stores must incur some costs in getting manufacturers to label products with unique names; car dealers could presumably reduce their sales staff if they used posted prices; and banks must incur customer service costs to deal with questions and complaints that arise from their complicated fee structures.

In this section we explore such applications by considering the case when the obfuscation cost $c(t)$ that firms must incur in order to raise the time cost of search to $t$ is not identically zero. Obfuscation costs will naturally eliminate much of the indeterminacy of the costless model, as firms will not want to do any more obfuscation than is necessary to limit consumer search to its equilibrium level. Making obfuscation costly thereby creates more deterministic relationships between price and obfuscation in the cross-section, which we will characterize.

The two subsections do this in two different ways. The first is an equilibrium refinements approach: we simply posit that it is reasonable to expect that equilibria would satisfy a given minimal obfuscation condition and characterize equilibria satisfying the refinement. The second takes the more standard approach of having firms maximize a profit function that includes explicit obfuscation costs.

\subsection{Slightly costly obfuscation}

In this section we examine the implications of costly obfuscation in a somewhat nonstandard way: rather than explicitly introducing a small obfuscation cost, we refine the set of equilibria of the costless obfuscation model. We do this because we feel that this is a parsimonious way to bring out our several insights. But it also has some drawbacks: one can debate the refinement; and the equilibrium existence theorem we can provide requires more convexity of the $g$ function than we would like. Readers who will be too bothered by these drawbacks can skip to the next section which derives results similar to some of the results of this section using a more standard approach.

We will call a nontrivial NE of that model a minimal obfuscation NE if conditional on each realization of firm $i$ 's price, firm $i$ always chooses the lowest obfuscation level that yields the same profit, i.e. $(p, t)$ is not in the support of firm $i$ 's mixed strategy if there exists a $\left(p, t^{\prime}\right)$ with $t^{\prime}<t$ that yields the same profit. This could be roughly motivated by thinking about obfuscation costs that exist but are lexicographically less important than 
profits, but our refinement does not impose all restrictions that this would imply. ${ }^{14}$ We characterize the price-obfuscation relationship in these equilibria, and also gain insight into what obfuscation levels are feasible in any nontrivial NE.

As a first step in the analysis, we provide conditions for the existence of a minimal obfuscation NE.

Proposition 7 Assume that $\lim _{t \rightarrow \infty} g^{\prime}(t)=\infty$. Let $\tilde{t}$ be such that

$$
g(2 \tau+\tilde{t})-g(\tau+\tilde{t})=V\left(p^{m}\right)
$$

If $g(\tau+\tilde{t}) \leq V(\infty)$, then there exists a minimal obfuscation $N E$.

The proof is presented in the Appendix. The main idea is that a symmetric mixed strategy profile is a minimal obfuscation $\mathrm{NE}$ if and only if firms choose prices from $\left[\underline{p}, p^{m}\right]$ according to $F(p)$ and choose an obfuscation level $t(p)$ conditional on each price realization satisfying

$$
t\left(p_{i}\right)=\inf \left\{t_{i} \in \mathbb{R}^{+}: \mathbb{E}_{p}\left[g\left(2 \tau+t(p)+t_{i}\right)-g\left(\tau+t_{i}\right)\right] \geq V\left(p_{i}\right)\right\}
$$

We show the existence of such a function $t$ by applying Schauder's Fixed Point Theorem to the operator given by

$$
T(t(p))\left(p_{i}\right)=\inf \left\{t_{i} \in \mathbb{R}^{+}: \mathbb{E}_{p}\left[g\left(2 \tau+t(p)+t_{i}\right)-g\left(\tau+t_{i}\right)\right] \geq V\left(p_{i}\right)\right\}
$$

Note that the assumption that $\lim _{t \rightarrow \infty} g^{\prime}(t)=\infty$ is needed to guarantee that the operator $T$ is well-defined. Similar assumptions are made in many literatures in which some function is convex, but we regard the assumption as unappealing because part of what makes our basic argument striking is that it only requires that $g$ be slightly convex. Note also that we had avoided making any such assumption in our earlier results. Indeed, in Lemma 3 and Proposition 3 among other places our arguments could have been substantially simpler if we had been willing to make such an assumption. The condition $g(\tau+\tilde{t}) \leq V(\infty)$ says that costly searchers enter the market when firms obfuscate to the point where a costly searcher

\footnotetext{
${ }^{14}$ It is not equivalent to the lexicographic formulation because we are only requiring that obfuscation be minimal conditional on the price the firm has chosen, not also that the firm only mix over prices which are compatible with doing the least possible obfuscation.
} 
is indifferent between searching and stopping conditional on observing $p=p^{m}$ and $t=\tilde{t}$ when she expects all other firms to set $t=0$. This rather strong assumption is necessary to ensure that costly searchers enter when obfuscation levels are given by a fixed point of $T$.

The characterization of a minimal obfuscation NE given by (4) makes it easy to understand the price-obfuscation relationship. There will be a subinterval $\left[\underline{p}, p^{\prime}\right]$ on which $t(p)$ is identically zero, because even the lowest possible search costs, $g(2 \tau)-g(\tau)$, will be sufficient to deter consumers who find prices sufficiently close to $\underline{p}$ from getting a second price quote. At higher price levels, obfuscation levels are positive and strictly increasing in $p$. At the highest prices, obfuscation levels must be above the minimum possible uniform obfuscation level $t^{u}$ identified in the previous section. ${ }^{15}$ Note that this increasing pattern contrasts with the predictions of the model of Wilson (2008). In Wilson's two-firm model, the firm that obfuscates chooses prices from a distribution that is lower than the distribution from which the non-obfuscating firm chooses its prices.

Another benefit of having characterized and shown the existence of minimal obfuscation $\mathrm{NE}$ is that we gain additional insight into what levels of obfuscation are possible in the costless model. In the previous section, we characterized the lowest and highest possible levels of obfuscation in a NE in which all firms choose the same obfuscation level. We note here that the lowest $t^{u}$ which is possible in a NE with price-independent obfuscation is not a lower bound on the expected obfuscation in any NE. Indeed, we show that the average obfuscation level in any minimal obfuscation NE - which must exist by Proposition 7-is lower.

Proposition 8 Let $\underline{t}^{u}$ be the minimum obfuscation level such that there exists a nontrivial NE in which all firm use obfuscation level $\underline{t}^{u}$. This $\underline{t}^{u}$ is given by

$$
g\left(2\left(\tau+\underline{t}^{u}\right)\right)-g\left(\tau+\underline{t}^{u}\right)=\int_{\underline{p}}^{p^{m}} D(p) F(p) d p
$$

Suppose that $t^{*}(p)$ is the obfuscation function from a minimal obfuscation NE. Let

$$
\bar{t}^{*} \equiv \int_{\underline{p}}^{p^{m}} t^{*}(p) f(p) d p
$$

\footnotetext{
${ }^{15}$ This latter fact is actually shown in the proof of Proposition 8 below.
} 
be the average obfuscation level in this equilibrium. Then $\bar{t}^{*}<\underline{t}^{u}$.

Proof. We first claim that $t^{*}\left(p^{m}\right)>\underline{t}^{u}$. For suppose, towards a contradiction, that $t^{*}\left(p^{m}\right) \leq \underline{t}^{u}$. Then

$$
\mathbb{E}_{p}\left[g\left(2 \tau+t^{*}\left(p^{m}\right)+t^{*}(p)\right)-g\left(\tau+t^{*}\left(p^{m}\right)\right)\right]<g\left(2 \tau+t^{*}\left(p^{m}\right)+\underline{t}^{u}\right)-g\left(\tau+t^{*}\left(p^{m}\right)\right)
$$

as $t^{*}(p) \leq \underline{t}^{u}$ for all $p$ with strict inequality on a set of positive measure (since $t^{*}(p)$ is strictly increasing in $p$ in a neighborhood of $p^{m}$ ). Convexity of $g$ gives

$$
g\left(2 \tau+t^{*}\left(p^{m}\right)+\underline{t}^{u}\right)-g\left(\tau+t^{*}\left(p^{m}\right)\right) \leq g\left(2\left(\tau+\underline{t}^{u}\right)\right)-g\left(\tau+\underline{t}^{u}\right)
$$

and $g\left(2\left(\tau+\underline{t}^{u}\right)\right)-g\left(\tau+\underline{t}^{u}\right)=V\left(p^{m}\right)$, so combining the inequalities yields

$$
\mathbb{E}_{p}\left[g\left(2 \tau+t^{*}\left(p^{m}\right)+t^{*}(p)\right)-g\left(\tau+t^{*}\left(p^{m}\right)\right)\right]<V\left(p^{m}\right)
$$

contradicting the hypothesis that all costly searchers buy after at most one search when obfuscation levels are given by $t^{*}(p)$.

Now, arguing by contradiction once again, suppose that $\bar{t}^{*} \geq \underline{t}^{u}$. Then

$$
\begin{aligned}
\mathbb{E}_{p}\left[g\left(2 \tau+t^{*}\left(p^{m}\right)+t^{*}(p)\right)-g\left(\tau+t^{*}\left(p^{m}\right)\right)\right] & >g\left(2 \tau+t^{*}\left(p^{m}\right)+\bar{t}^{*}\right)-g\left(\tau+t^{*}\left(p^{m}\right)\right) \\
& \geq g\left(2 \tau+t^{*}\left(p^{m}\right)+\underline{t}^{u}\right)-g\left(\tau+t^{*}\left(p^{m}\right)\right) \\
& >g\left(2\left(\tau+t^{u}\right)\right)-g\left(\tau+\underline{t}^{u}\right)=V\left(p^{m}\right)
\end{aligned}
$$

contradicting the requirement that the firm setting price $p^{m}$ cannot cut its obfuscation level and still sell to costly searchers when obfuscation levels are given by $t^{*}(p)$. Note that the first and third inequalities above follow from strict convexity of $g$, while the second simply uses that $g$ is increasing.

The highest level of obfuscation that is possible in a NE in which all firms choose the same obfuscation level and consumers enter (as identified by equation (3) in the previous section) can easily be seen to be a tight upper bound on what is possible in any NE.

Proposition 9 Let $\bar{t}$ be the average obfuscation level in any nontrivial NE. Then $\bar{t} \leq \bar{t}^{u}$, where $\bar{t}^{u}$ is the solution to

$$
g\left(\tau+\bar{t}^{u}\right)=\int_{\underline{p}}^{\infty} D(p) F(p) d p
$$


Proof. If costly searchers enter, then

$$
\mathbb{E}[g(\tau+t)] \leq \int_{\underline{p}}^{\infty} D(p) F(p) d p
$$

where the expectation on the left side is over the marginal distribution induced by firms' mixed strategies. The convexity of $g$ implies that $g(\tau+\bar{t}) \leq \mathbb{E}[g(\tau+t)] \leq g\left(\tau+\bar{t}^{u}\right)$, so $\bar{t} \leq \bar{t}^{u}$.

Thus, considering NE in which obfuscation levels are not constant and price-independent shows that there may exist nontrivial NE with less obfuscation than those described in Section 3, though not with more obfuscation.

\subsection{Truly costly obfuscation}

In this section we consider the impact of obfuscation costs in a more standard way. Specifically, we assume that a firm must pay a cost $c(t)$ to set obfuscation level $t$, where $c(0)=0$ and $c$ is strictly increasing and convex. We provide some results analogous to those of the previous section and also note some ways in which the equilibria change.

Our first observation is that whether the equilibrium differs from that of the costlessobfuscation model depends on the search cost function, rather than on the obfuscation cost function.

Proposition 10 Let $V(p)$ be the consumer benefit from search assuming that prices are distributed as in the nontrivial NE of the costless-obfuscation model.

(a) If $g(\tau)<V(\infty)$ and $g(2 \tau)-g(\tau)>V\left(p^{m}\right)$, then the model with costly obfuscation has a unique nontrivial NE, in which no obfuscation occurs and the equilibrium price distribution coincides with that of the costless-obfuscation model.

(b) If $g(2 \tau)-g(\tau)<V\left(p^{m}\right)$, then any nontrivial $N E$ of the costly obfuscation model must have prices distributed on an interval $[\underline{p}, \bar{p}]$ with $\bar{p}$ strictly less than $p^{m}$.

Proof. For part (a) note that the the conditions on $g$ imply that consumers will search once and will not search a second time if firms mix over prices as they do in the equilibrium of the costless obfuscation model. Hence, the same calculations as for the costless obfuscation model apply and show that there is no profitable deviation that involves zero obfuscation. 
Deviations that involve positive obfuscation are also not profitable, because they cannot lead to making greater sales (for any price weakly less than $p^{m}$ ) and any obfuscation costs incurred will only reduce profits.

For part (b), note first that Lemmas 1 and 2 imply that prices are distributed according to some atomless distribution $F^{*}$ on some interval $[\underline{p}, \bar{p}]$ with $\underline{p}>c$. We must have $\bar{p} \leq p^{m}$ because any price greater than $p>p^{m}$ is dominated by setting $p=p^{m}$ and using the same obfuscation level. We claim that firms setting prices $p_{0}$ in a neighborhood of $\bar{p}$ must sell to all consumers who visit them first. To see this, first note that if they did not sell to any consumers who visit them first then they earn arbitrarily small profits (which is impossible, since all firms must earn the same, strictly positive level of profits in any NE), as consumers purchase only from the lowest-price firm they visit, and firms with prices close to $p^{m}$ have a vanishingly small probability of being the lowest-price firm visited by any consumer who has searched more than once. And if such a firm sells to only some of those consumers that visit it first, it can cut prices by an arbitrarily small amount and sell to all of these consumers, yielding a discrete gain in profits. Such firms must also be choosing positive obfuscation levels satisfying

$$
\mathbb{E}_{p}\left[g\left(2 \tau+t(p)+t\left(p_{0}\right)\right)\right]-g\left(\tau+t\left(p_{0}\right)\right)=\int_{\underline{p}}^{p_{0}} D(p) F^{*}(p) d p .
$$

where $F^{*}(p)$ is the equilibrium price distribution. Indeed, they cannot set lower obfuscation levels because such levels would cause consumers to search again and result in these firms earning near-zero profits; and level of $t\left(p_{0}\right)$ implied by this equation cannot be negative because then no firms would engage in obfuscation and $F$ would be the distribution from the previous section, which we have assumed to induce further search. Hence, for all $p_{0}$ just below $\bar{p}$ a first-order approximation to the profit function is

$$
\left.R\left(p_{0}\right) \frac{1-\mu}{N}-c\left(t\left(p_{0}\right)\right)\right)
$$

This expression is strictly decreasing for $p_{0}$ near $p^{m}$, because $R^{\prime}\left(p^{m}\right)=0$ and the cost term has a nonzero derivative. Hence, $\bar{p}$ cannot be equal to $p^{m}$.

The main difference between the equilibrium of the costless obfuscation model and what part (b) notes is possible with costly obfuscation is that costly obfuscation implies 
that $\bar{p}<p^{m}$. The last line of the proof contains all the intuition: prices must be bounded away from the monopoly price because if $\bar{p}=p^{m}$ then there is a first-order gain from reducing the level of obfuscation necessary to deter consumer search, and no first-order loss in per customer profits.

Note also that in the course of the proof of part (b) we have shown that some obfuscation must occur in any nontrivial NE in that case: firms with prices near $\bar{p}$ must engage in obfuscation because otherwise consumers will conduct a second search and the firms will earn zero profits.

In our slightly costly model we had a simple expression for the obfuscation level of each firm. The analog to that conclusion is:

Proposition 11 In any nontrivial NE, a firm that sets price $p_{0}$ must set obfuscation level $t\left(p_{0}\right)$ such that

$$
\mathbb{E}_{p}\left[g\left(2 \tau+t(p)+t\left(p_{0}\right)\right)\right]-g\left(\tau+t\left(p_{0}\right)\right) \leq \int_{\underline{p}}^{p_{0}} D(p) F^{*}(p) d p .
$$

The proof of this proposition is immediate: there is no point in doing any more obfuscation than is necessary to deter further consumer search. What is more noteworthy is what is not in the proposition: we do not claim that the equation holds with equality whenever obfuscation is necessary to deter further consumer search. The reason is that firms who have chosen low prices may choose to do little or no obfuscation even though this may induce consumers to conduct a second search - unlike in the textbook model with constant search costs, consumers in our model have convex search costs, so they may search just one more time and then return to the first firm with high probability. We think this adds realism to the model and it results in some interesting patterns. For example, search can be nonmonotone in prices with consumers who find very low prices purchasing right away, consumers who find slightly higher prices searching a second time before giving up, and consumers who find the highest prices buying immediately because the high-priced firms engage in sufficient obfuscation to use up the time consumers were willing to spend searching.

An unfortunate consequence of this feature is that it makes characterizing equilibrium strategies cumbersome: demand is a complicated function of the vector of prices and there 
is no neat expression for the equilibrium $F^{*}$ as there is when consumers search exactly once in equilibrium. Nevertheless, several important properties of the distribution of equilibrium obfuscation levels carry over from the slightly costly obfuscation case. As before, there is an interval of positive length $\left[\underline{p}, p^{\prime}\right]$ on which firms set zero obfuscation, as the exogenous component of search costs is sufficient to deter a second search for consumers who first observe a price sufficiently close to $\underline{p}$. And there is another interval of positive length $\left[p^{\prime \prime}, \bar{p}\right]$ on which firms set obfuscation such that the inequality in Proposition 11 holds with equality $^{16}$, so that obfuscation is increasing in price. Furthermore, a firm that sets price $\bar{p}$ must also set the highest obfuscation level; since such a firm sells to costly searchers that visit it with probability one, any other firm need not obfuscate so much to sell to its customers with probability one, and beyond this point there is no incentive to obfuscate. The only potential difference in the pattern of equilibrium obfuscation across firms between the slightly costly and truly costly obfuscation models, then, is that in the truly costly obfuscation model the union of the intervals $\left[\underline{p}, p^{\prime}\right]$ and $\left[p^{\prime \prime}, \bar{p}\right]$ need not equal $[\underline{p}, \bar{p}]$. That is, in both models the lowest-priced firms do not use obfuscation and the highest-priced firms use the most obfuscation, but only in the slightly costly obfuscation model can we rule out nonmonotonicity in the obfuscation level.

Indeed, it seems quite possible that one could construct an example with nonmonotone obfuscation if obfuscation is truly costly for firms. We sketch such an example here: Suppose that $c(t)$ is very small up to a point $t^{*}$ and increases rapidly thereafter, and that that $g(t)$ and $\tau$ are such that no consumer is ever willing to search three times but a consumer may search a second time if the obfuscation she encountered on her first search was sufficiently low. We conjecture that there exist parameters such that firms with the lowest prices set $t=0$ and sell to consumers on their first search, firms with slightly higher prices set $t \in\left(0, t^{*}\right)$ and sell to consumers on their first search (since doing so is not very costly), firms with intermediate prices set $t=0$ and sell to consumers on their second search (since setting high enough obfuscation to sell to consumers on their first search would be very expensive), firms with even higher prices set $t \in\left(0, t^{*}\right)$ and sell to consumers on their second search, and firms with the highest prices set $t \geq t^{*}$ and sell to consumers on their first search (since

\footnotetext{
${ }^{16}$ This is shown in the proof of Proposition 10.
} 
such firms would hardly ever sell if they did not sell to consumers on their first search).

\section{$5 \quad$ A Signal-Jamming Model}

In this section we explore an alternate mechanism through which obfuscation can affect consumer search. At the broadest level, our signal-jamming model is another way to reach the conclusions that firms obfuscate in equilibrium and that this affects the equilibrium price distribution. But the mechanics of the argument are different, and this leads to some differences in the results. Among these are an "excess obfuscation" problem that can prevent the existence of equilibria with consumer entry, a different selection among the possible equilibria of Stahl's model, and the presence of effects that can change comparative statics results. In particular, there is no longer a unique equilibrium price distribution, and the set of equilibrium price distributions depends on parameters in interesting ways.

The basic idea behind our signal-jamming mechanism is straightforward. We assume that search costs are linear rather than convex. But we assume that there is uncertainty about the exogenous component of search costs. Consumers learn about this component from their first shopping experience, so obfuscation raises consumer expectations about the search costs they will incur on future searches. This mechanism seems plausible for many applications. For example, if a home-improvement contractor spends a long time with a consumer discussing details about the job and takes a long time to prepare and submit his or her bid, then it seems plausible that consumers will expect that getting a second bid will entail similar time costs.

Formally, we assume that there is an underlying parameter $\theta$ with expectation zero distributed with continuous, strictly positive density $h(\theta)$ on $[\underline{\theta}, \infty)$ with $\underline{\theta}>-\tau$, such that it costs a consumer $\tilde{t}_{i}=\tau+\theta+t_{i}$ to visit a firm that sets obfuscation level $t_{i}$. We assume that when a consumer visits firm $i$, she observes only $\tilde{t}_{i}$ and $p_{i}$, so that she must draw inferences about $\theta$ in equilibrium. The timing of the game is almost as before. The one amendment is that we assume that $\theta$ is drawn once and for all at the beginning of the game and is unobserved by both firms and consumers. ${ }^{17}$

We focus in this section on costless obfuscation. As before, this lets us bring out our

\footnotetext{
${ }^{17}$ We assume that firms do not observe $\theta$ to avoid a large multiplicity of signaling equilibria.
} 
main observations most simply. We also restrict our attention to strategies for firms which do not mix over obfuscation levels for a given price. That is, we consider equilibria in which there exists a function $t^{*}(p)$ such that the support of firms' mixed strategies is contained in the set of ordered pairs $(p, t)$ with $t=t^{*}(p)$. The import of the assumption is that equilibrium implies that consumers believe with probability one that $\theta=\hat{\theta} \equiv \tilde{t}-\left(\tau+t^{*}(p)\right)$ after observing total search cost $\tilde{t}$ and price $p^{18}$. The only exception to this, of course, is if the observed $(p, t)$ is inconsistent with equilibrium, which can happen if $p$ not in the support of the equilibrium price distribution or if $\tilde{t}<\tau+t^{*}(p)+\underline{\theta}$. We do not restrict beliefs in this case, but will, of course, assume that consumers act rationally given some belief about $\theta$. Throughout this section we'll write PBE as a shorthand for "symmetric Perfect Bayesian Equilibrium in which firms do not mix over obfuscation levels for a given price."

\subsection{Consumer search behavior}

In this section we note that consumer search behavior in the signal-jamming model is similar to search behavior in our previous model. First, we have a straightforward application of standard results.

Proposition 12 In any PBE, a costly searcher searches for the first time if $\tau+\mathbb{E}[t(p)]<$ $V(\infty)$ and continues to search if $\tau+\hat{\theta}+\mathbb{E}[t(p)]<V\left(p_{0}\right)$ and there are previously unsearched firms remaining. Conversely, a costly searcher does not search for the first time if $\tau+$ $\mathbb{E}[t(p)]>V(\infty)$ and stops searching if $\tau+\hat{\theta}+\mathbb{E}[t(p)]>V\left(p_{0}\right)$.

Next, we observe that costless search implies that costly searchers search at most once in equilibrium, as in the previous model.

Proposition 13 In any PBE of the signal-jamming model with costless obfuscation, all costly searchers search at most once.

Proof. Fix a PBE obfuscation strategy $t(p)$ and suppose that a firm does not sell to all costly searchers that visit it first when it sets price $p$ and obfuscation level $t(p)$. A consumer

\footnotetext{
${ }^{18}$ Without this result, the consumer search problem would become much more complicated, as consumers would have an incentive to search multiple times in order to learn more about $\theta$.
} 
who first visits a firm with price $p$ will buy if $\tilde{t}-t(p)+\mathbb{E}[t(p)]>V(p)$, since in equilibrium the consumer infers that $\theta=\tilde{t}-(t(p)+\tau)$ whenever she observes total search cost $\tilde{t}$ and price $p$. Therefore, a firm can always induce those consumers who visit it first to buy with probability 1 by setting $t>t(p)+V(p)-\mathbb{E}[t(p)]$. By Proposition 12, this maximizes the market share of the firm, so there cannot be a PBE in which a firm does not sell to all consumers who visit it first.

The fact that consumers search at most once will again allow us to provide simple, closed-form expressions for the possible PBE price distributions. It is the primary place where we use the assumption that the distribution of $\theta$ is unbounded. If $\theta$ is bounded, it may be that consumers sometimes search multiple times.

\subsection{Equilibrium prices and obfuscation}

The fact that equilibrium consumer search is similar in the two models will lead to similarities in the equilibrium outcomes between the signal-jamming model and our earlier convex search cost model. But the way in which firms can use obfuscation to influence consumer behavior differs across the two models. In this section we bring out the differences in what firms can do and note the effect this has on equilibrium outcomes.

We first note that there are more serious obstacles to the existence of equilibria in which $\bar{p}=p^{m}$ in the signal-jamming model.

Proposition 14 Let $V\left(p_{0}\right) \equiv \int_{\underline{p}}^{p^{0}} D(p) F(p) d p$ be the value of search when prices are drawn from the NE of the model with convex search costs and costless obfuscation described in Proposition 3. The signal-jamming model has a nontrivial PBE with a price distribution having support $\left[\underline{p}, p^{m}\right]$ if and only if $\tau \leq V(\infty)$ and $\int_{p^{m}}^{\infty} D(p) d p \geq-\underline{\theta}$.

Proof. Because consumers search once, such an equilibrium must have prices distributed as in Proposition 3. Hence, $V$ is the value to consumers of an additional search. Let $\bar{t}$ be the average level of obfuscation. Consumers will search exactly once if and only if entry and stopping conditions hold. $\tau+\bar{t} \leq V(\infty)$ is needed to allow consumers to conduct an initial search. And $\tau+\underline{\theta}+\bar{t} \geq V\left(p^{m}\right)$ is needed to prevent consumers who find price $p^{m}$ and discover that $\theta=\underline{\theta}$ from searching a second time. There exists a value of $\bar{t}$ satisfying 
both constraints if and only if $V(\infty) \geq V\left(p^{m}\right)-\underline{\theta}$ and $\tau \leq V(\infty) .{ }^{19}$ The first of these conditions is equivalent to

$$
-\underline{\theta} \leq V(\infty)-V\left(p^{m}\right)=\int_{p^{m}}^{\infty} D(p) d p .
$$

Finally, if prices are distributed on $\left[\underline{p}, p^{m}\right]$ as in Proposition 3 and all consumers purchase from the first store they visit, firms have no profitable deviation, since they are indifferent between charging any two prices in $\left[\underline{p}, p^{m}\right]$ and earn lower profits from charging outside this interval.

The first condition in the Proposition, $\tau \leq V(\infty)$, is simply the requirement that the exogenous search costs are not high enough to prevent consumers from searching at least once. It is analogous to the sole condition required for the existence of a nontrivial NE in the convex search cost model. The second condition, $\int_{p^{m}}^{\infty} D(p) d p \geq-\underline{\theta}$, is an additional restriction requiring that the consumer surplus from purchasing at the monopoly price also be sufficiently large relative to the uncertainty about $\theta$. This reflects that there is what one can think of as "excess obfuscation" in the signal-jamming model with costless obfuscation. In equilibrium, firms must obfuscate to the point where consumers will not want to search again even when the exogenous component of search costs turns out to take on its lowest possible value. This implies that, with probability one, the average obfuscation level in any PBE is higher than the minimal average obfuscation level needed to keep all costly searchers from conducting a second search, conditional on $\theta$. This excess obfuscation makes it harder to sustain equilibria in which consumers search, as the lowest $\bar{t}$ such that $\tau+\underline{\theta}+\bar{t} \geq V\left(p^{m}\right)$ (the stopping condition) may be so high that $\tau+\bar{t}>V(\infty)$ (the negation of the entry condition), precluding costly searcher entry. To put this another way, in the convex search costs model we could always simultaneously satisfy the entry and stopping conditions as long as $g(\tau)$ was less than $V(\infty)$. Now, the difference between $\underline{\theta}$ and $\mathbb{E}[\theta]$ drives a wedge between the entry and stopping conditions, so we may not be able to satisfy them both simultaneously.

We now provide a complete characterization of the nontrivial PBE of the signal-jamming

\footnotetext{
${ }^{19}$ To see this, first note that, if these two conditions hold, taking $\bar{t}=V(\infty)-\tau$ satisfies the constraints. If the constraints hold, $\tau \leq V(\infty)$ is immediate and $V(\infty) \geq V\left(p^{m}\right)-\underline{\theta}$ follows by subtracting the second constraint from the first.
} 
model. Because consumers search once in equilibrium, the distribution of prices must look like the price distribution in Stahl's model for some search cost. But the selection turns out to be different from that of our previous model. In the convex-search-cost model, the key deviation that prevented almost all of these distributions from being equilibria was that a firm could charge a price slightly above $\bar{p}$ and obfuscate slightly more so that consumers would not search again. This constraint on the PBE set no longer exists in the signaljamming model: the above deviation is a deviation to an out-of-equilibrium price, so the firm cannot necessarily induce any consumers to have the beliefs about $\theta$ that it would like them to have after such deviations. In particular, a consumer who observes a price above $\bar{p}$ may believe that the firm that set this price also set very high obfuscation, which would lead her to believe that $\theta$ is very low. So, whereas in the convex costs model $\bar{p}$ was fixed at $p^{m}$, in this model the range of possible values for $\bar{p}$ is determined by the entry and stopping conditions.

It turns out that we often get an incomplete selection from the equilibria of Stahl's model. Many price distributions may be possible.

Proposition 15 For every $p^{*} \in\left[0, p^{m}\right)$, there is a nontrivial PBE price distribution $F_{p^{*}}(p)$ of the signal-jamming model with costless obfuscation with the supremum of the support of $F_{p^{*}}(p)$ equal to $p^{*}$ only if

$$
F_{p^{*}}(p)=1-\left[\left(\frac{1-\mu}{N \mu}\right)\left(\frac{R\left(p^{*}\right)}{R(p)}-1\right)\right]^{\frac{1}{N-1}}
$$

for all $p \in\left[\underline{p}^{*}, p^{*}\right]$, where $\underline{p}^{*}$ is given by $R\left(\underline{p}^{*}\right)=\left[\frac{1-\mu}{1+(N-1) \mu}\right] R\left(p^{*}\right)$. Such a PBE exists if and only if $\int_{\underline{p}^{*}}^{p^{*}} D(p) F_{p^{*}}(p) d p \geq \tau+\underline{\theta}$ and $\int_{p^{*}}^{\infty} D(p) d p \geq-\underline{\theta}$. Furthermore, some nontrivial $P B E$ exists if $\tau \leq \int_{0}^{\infty} D(p) F_{p^{m}}(p) d p$.

The proof is given in the Appendix. The main idea is to initially follow steps as in our earlier characterizations and then introduce the new constraints as in the previous proposition. The principle new intuition in this result as compared to Proposition 14 is that if $p^{*}<p^{m}$ firms are tempted to price above $p^{*}$, so in equilibrium consumers must be willing to undertake a second search after observing $p>p^{*}$ when they believe that $\theta$ is as low as possible. A necessary condition for this is $\int_{\underline{p}^{*}}^{p^{*}} D(p) F_{p^{*}}(p) d p \geq \tau+\underline{\theta}$, and Proposition 
15 shows that this condition and the requirement that the excess obfuscation problem is not too severe are jointly sufficient.

The main difference in the outcome between the signal-jamming model and the convex costs model is that in the signal-jamming model there may be PBE with $\bar{p}<p^{m}$. Indeed there will be parameter values for which such PBE exist while equilibria with $\bar{p}=p^{m}$ do not. To construct equilibria with $\bar{p}<p^{m}$, one simply assumes that consumers who see a price greater than $\bar{p}$ believe that $\theta$ is low. Note that the necessary and sufficient conditions for the existence of an equilibrium with $\bar{p}=p^{*}$ tend to hold for intermediate values of $p^{*}$. It is easy to see why $p^{*}$ cannot be too low in equilibrium: if $p^{*}$ is close to 0 , firms would deviate to price slightly above $p^{*}$ and costly searchers would purchase even if they believe that $\theta$ is low. The argument for why $p^{*}$ cannot be too high in equilibrium is more subtle and comes from the excess obfuscation effect. The idea is that lower prices correspond to higher consumer welfare and a larger gap between consumer welfare and the benefit of a second search conditional on observing $p^{*}$ and $\underline{\theta}$, which makes it more likely that the excess obfuscation needed to satisfy the stopping condition is not so great as to violate the entry condition.

Finally, observe that even though Proposition 15 places both lower and upper bounds on equilibria $p^{*}$, the last sentence of the proposition shows that existence of some nontrivial PBE is guaranteed under conditions identical to those in the convex search cost model.

\subsection{Analysis and comparative statics}

We provide some observations about the emergence of equilibrium obfuscation and its effect on prices, profits and consumer welfare, as we did in the convex-costs model. The results here are slightly more subtle due to the range of PBE described in Proposition 15, but two important ideas - that obfuscation occurs in equilibrium, and that obfuscation offsets changes in the exogenous component of consumer search costs - continue to come through. In addition, we show that prices actually fall as the excess obfuscation problem described above becomes more severe, i.e. as $\underline{\theta}$ decreases, but that nonetheless consumer welfare falls in response to this as well.

Our first corollary provides conditions sufficient to imply that some obfuscation must 
take place. As in the convex search cost model, obfuscation must occur in equilibrium unless exogenous search costs are so high that the stopping condition is satisfied with zero obfuscation. We omit the proof, which is analogous to the proof of Corollary 1 .

Corollary 3 In any nontrivial PBE with $\bar{p}=p^{*}$ and $\underline{\theta}+\tau<\int_{\underline{p}^{*}}^{p^{*}} D(p) F_{p^{*}}(p) d p$, where $F_{p^{*}}$ is given by equation (5), some firms set $t>0$.

To simplify our comparative statics analysis in this section, we henceforth impose the following assumption: ${ }^{20}$

Assumption $1 \int_{\underline{p}^{*}}^{p^{*}} D(p) F_{p^{*}}(p) d p$ is increasing in $p^{*}$ for $p^{*}<p^{m}$.

We first show that the results on the effect of changes in the exogenous component of consumer search costs on average equilibrium obfuscation and consumer welfare in this model are qualitatively the same as in the convex search costs model with uniform obfuscation. That is, decreases in $\tau$ lead to increases in both average obfuscation and consumer welfare.

Proposition 16 The set of nontrivial PBE average obfuscation levels is decreasing in $\tau$ in the strong set order. Furthermore, for any $p^{*}$, the set of nontrivial PBE average obfuscation levels corresponding to PBE with $\bar{p}=p^{*}$ is decreasing in $\tau$ in the strong set order.

Proposition 17 The set of nontrivial PBE values of the costly searchers' welfare is decreasing in $\tau$ in the strong set order.

Proofs of these propositions are given in the appendix. Each builds off the observation that in any nontrivial PBE with the upper bound of the price distribution equal to $p^{*}<p^{m}$, the average obfuscation level must be $\int_{\underline{p}^{*}}^{p^{*}} D(p) F_{p^{*}}(p) d p-\underline{\theta}-\tau$ : consumers must not want to search again if the price is $p^{*}$ and they find out that $\theta=\underline{\theta}$ and they must be willing to search again if the firm deviates to any $p>p^{*}$. Proposition 16 reflects that this obfuscation level is decreasing in $\tau$ and that the set of equilibrium obfuscation levels in equilibria with upper

\footnotetext{
${ }^{20}$ This assumption is implied by Assumption C in Stahl (1989), which is the same as the "Revenue Condition" in Stahl (1996). As Stahl (1996) points out, this condition holds "for all concave (and linear) demand functions, as well as many convex demand functions."
} 
bound $p^{m}$ is also decreasing in $\tau$. An intuition for Proposition 17 is that, holding prices fixed, the direct effect of increasing $\tau$ outweighs the indirect effect of reduced obfuscation, as in Proposition 5. And, under Assumption 1, the set of PBE price distributions is increasing in $\tau$ in the strong set order, so the direct effect cannot be offset by favorable changes in the price distribution.

While we find Propositions 16 and 17 noteworthy, they do not depend on the signaljamming structure of our model. In particular, both of these results hold in the alternative model where $\theta$ is deterministic and search costs are linear. ${ }^{21}$ The main consequence of the signal-jamming structure of the model for comparative statics, then, is the excess obfuscation effect. To derive comparative statics on this effect (Propositions 18 and 19) we consider changes in $\underline{\theta}$, holding $\mathbb{E}[\theta]$ constant at zero.

Recalling that an increase in $\underline{\theta}$ corresponds to a decrease in the severity of the excess obfuscation problem, Proposition 18 shows that an increase in the severity of this problem leads to a decrease in prices, which is perhaps a surprising result. The intuition here is that the excess obfuscation problem rules out equilibria with high prices, as per the discussion following Proposition 15, so increasing the severity of the excess obfuscation problem eliminates equilibria with the highest prices. Proposition 19 shows that, if all equilibria have $\bar{p}<p^{m}$ this effect cannot overturn the direct welfare costs to consumers of an increase in excess obfuscation, because excess obfuscation leads to lower prices only by making consumers sufficiently worse off that they refuse to enter when prices are high. The assumption that equilibria with $\bar{p}=p^{m}$ do not exist is needed for this result because equilibria with $\bar{p}=p^{m}$ can have very high obfuscation, since the only upper bound on obfuscation in this case is the entry condition, while equilibria with $\bar{p}<p^{m}$ must also have low enough obfuscation that firms are not tempted to deviate to prices slightly above $\bar{p}$, which makes comparing consumer welfare across equilibria with $\bar{p}<p^{m}$ and $\bar{p}=p^{m}$ difficult. So long as equilibria with $\bar{p}=p^{m}$ do not exist, then, Propositions 18 and 19 show that, while equilibrium requires that firms expropriate some of the additional surplus that comes with an increase in $\underline{\theta}$ through reduced reduced excess obfuscation, consumers are still better off after such a reduction in uncertainty, at least in the strong set order sense. In this

\footnotetext{
${ }^{21}$ However, firms would not have a strict incentive to obfuscate in such a model.
} 
sense increases in $\underline{\theta}$ are Pareto-improving, even though they make markets less competitive in that they raise equilibrium prices.

Proposition 18 The set of nontrivial PBE values of $\bar{p}$ is increasing in $\underline{\theta}$ in the strong sense order.

Proof. Recall that $p^{*}$ is a nontrivial PBE value of $\bar{p}$ if and only if $\int_{\underline{p}^{*}}^{p^{*}} D(p) F_{p^{*}}(p) d p \geq \tau+\underline{\theta}$ and $\int_{p^{*}}^{\infty} D(p) d p \geq-\underline{\theta}$. And $\int_{\underline{p}^{*}}^{p^{*}} D(p) F_{p^{*}}(p) d p$ is increasing in $p^{*}$ for $p^{*}<p^{m}$ by assumption, while $\int_{p^{*}}^{\infty} D(p) d p$ is decreasing in $p^{*}$, so an increase in $\underline{\theta}$ raises the lower bound on $\bar{p}$ given by the first inequality and raises the upper bound of $\bar{p}$ given by the second.

Proposition 19 Suppose that no PBE with $\bar{p}=p^{m}$ exist when the lower bound on $\theta$ equals $\underline{\theta}$ or $\underline{\theta}^{\prime}$ for some $\underline{\theta}^{\prime} \geq \underline{\theta}$. Then increasing the lower bound on $\theta$ from $\underline{\theta}$ to $\underline{\theta}^{\prime}$ increases the set of PBE values of consumer welfare in the strong set order.

Proof. Suppose that $\underline{\theta}^{\prime} \geq \underline{\theta}$ and $u^{\prime} \leq u$, where $u$ is a PBE value of consumer welfare with lower bound on $\theta$ given by $\underline{\theta}$ and $u^{\prime}$ is a PBE value of consumer welfare with this lower bound given by $\underline{\theta}^{\prime}$ Denote the upper bound of the price distribution yielding consumer welfare $u$ by $\bar{p}$ and denote the corresponding upper bound for $u^{\prime}$ by $\bar{p}^{\prime}$. We must show that $u^{\prime}$ is a PBE value when the lower bound is given by $\underline{\theta}$ and that $u$ is a PBE value when this bound is given by $\underline{\theta}^{\prime}$.

We have that $\bar{p}$ and $\bar{p}^{\prime}$ are both less than $p^{m}$, so the proof of Proposition 15 gives that $\int_{\underline{\bar{p}}}^{\bar{p}}=\tau+\underline{\theta}+\bar{t}$ and $\int_{\underline{\underline{p}}^{\prime}}^{\bar{p}^{\prime}}=\tau+\underline{\theta}+\bar{t}^{\prime}$, where $\bar{t}$ and $\bar{t}^{\prime}$ are average obfuscation levels corresponding to $\mathrm{PBE}$ with price upper bound $\bar{p}$ and welfare $u$, and price ceiling $\bar{p}^{\prime}$ and welfare $u^{\prime}$, respectively. Recall that $u=\int_{\underline{p}}^{\infty} F_{\bar{p}}(p) D(p) d p-\tau-\bar{t}$, so we have $u=\int_{\bar{p}}^{\infty} D(p) d p+\underline{\theta}$ and $u^{\prime}=\int_{\bar{p}^{\prime}}^{\infty} D(p) d p+\underline{\theta}^{\prime}$. Since $u^{\prime} \leq u$, this implies that $\bar{p}^{\prime} \geq \bar{p}$. Therefore, we have

$$
u^{\prime}=\int_{\bar{p}^{\prime}}^{\infty} D(p) d p+\underline{\theta}^{\prime} \leq u \leq \int_{\bar{p}}^{\infty} D(p) d p+\underline{\theta}^{\prime}
$$

The Intermediate Value Theorem them implies that there exists $p^{*} \in\left[\bar{p}, \bar{p}^{\prime}\right]$ such that $u=\int_{p^{*}}^{\infty} D(p) d p+\underline{\theta}^{\prime}$, which then implies that $u$ is a PBE value of consumer welfare when the lower bound on $\theta$ is given by $\underline{\theta}^{\prime}$ and the upper bound on $p$ is given by $p^{*}$. The argument for $u^{\prime}$ is similar. 
Finally, we remark that the mechanics of the model we have presented in this section differ from those of standard signal-jamming models in an important way: Usually, the marginal benefit to jamming the signal is positive in equilibrium, and the equilibrium quantity of signal-jamming is determined by marginal signal-jamming costs that increase in the quantity of signal jamming. In our model, however, the marginal cost of signaljamming is held constant at zero, so equilibrium requires that the marginal benefit of signal-jamming equal zero as well. This is what leads to Proposition 13 and the excess obfuscation effect; in equilibrium, search costs must be so high that consumers search only once even when they observe the highest possible realization of $p$ and the lowest possible realization of $\theta$. We believe that this model with costly obfuscation would look more like a standard signal-jamming model. That is, signal-jamming costs would be convex, so in equilibrium the marginal benefit of signal-jamming would remain positive, implying that firms that charged high prices would not sell to consumers when the realization of $\theta$ was low. We view the analysis of this kind of model as a promising avenue for future research, though the failure of Proposition 13 to hold in such a setting would naturally make simple expressions for the equilibrium price and obfuscation distributions unlikely.

\section{Conclusion}

In this paper we have explored obfuscation using two related models in which obfuscation is treated as an action that increases the amount of time that consumers must spend to learn a firm's price. In both cases, the key impact of such actions is that they lead consumers to behave as if future search costs will be higher. In the convex-costs model this is because obfuscation directly increases the incremental costs that consumer would incur to perform another search. In the signal jamming model there is no real effect on the future, but an informational linkage implies that increased obfuscation leads consumers to expect higher future search costs.

In both models, we show that obfuscation must occur in an equilibrium unless the exogenous component of consumer search costs is high enough that consumers are willing to purchase at the highest equilibrium price in the absence of obfuscation. And we show that obfuscation has the same qualitative impact on welfare. It is bad for consumers both because 
it directly imposes costs on them and because it leads to higher prices. The higher prices make obfuscation beneficial for firms, except in the case when excess obfuscation makes the market completely collapse. Note that obfuscation benefits all firms, not only those who engage in it; even transparent firms benefit from serving an obfuscation-rich market, as their customers are prevented from comparison-shopping by other firms' obfuscation.

The mechanics of our models are similar to those of Stahl (1989). In both cases obfuscation can be seen as selecting among the dispersed price equilibria of Stahl's model. In the convex-costs model, the selection is that obfuscation must be sufficiently high to result in an equilibrium price distribution that goes all the way up to the monopoly price. In the signal-jamming model, the constraints are that overall obfuscation levels must be sufficiently high so that consumers are willing to search once, but never more than once. This can leave a range of possible dispersed-price equilibria.

Our two models also have similar comparative statics implications. In both, equilibrium obfuscation adjusts to offset changes in the exogenous component of consumer search costs, though in equilibrium consumers still benefit from reductions in exogenous search costs and are hurt by increases in these costs. The signal-jamming model is also distinguished by the fact that it displays excessive obfuscation with probability one; prices fall as the severity of this problem increases, but consumer welfare decreases.

Our analysis suggests a number of interesting avenues for future research. Our characterizations of the costly obfuscation model are limited. In reality we feel that it takes a great deal of cleverness for firms to devise effective obfuscation schemes, which could make such schemes quite costly. Such costs would be natural candidates for explaining why realworld obfuscation is limited. For example, we noted that whereas our convex costs model with costless obfuscation predicts that obfuscation will completely offset any technological reduction in search costs, Ellison and Ellison (2009) report that search is still fairly effective for at least some consumers in the environment they study. Developing models of costly obfuscation that are more tractable than ours could be challenging, but could have rewards both from a theoretical and from an applied perspective.

Relatedly, an important constraint on real-world obfuscation may be that consumers will give up on a firm before learning its price. In some of our models our analyses can 
be justified by assuming that consumers beliefs are such that they expect the remaining duration of search to be short enough so that continuing with current firm is better than paying $\tau$ to start over. But in other cases correlations between $p$ and $t$ will prevent such justifications. And in in any case there may be interesting lessons to learn from thinking about models in which search is a real continuous time stopping problem rather than just a series of one or zero decisions about whether to visit each firm.

Finally, we note that there are more basic related areas of search theory that have not been fully explored. We mentioned that assuming that search costs are convex rather than constant can create an environment in which search strategies are more interesting and realistic, with different consumers searching different number of times. Stahl (1996) explores one way to get such behavior by adding heterogeneous search costs, but the convex cost model could be a valuable complement and provide additional insights. 


\section{Appendix}

Proof of Proposition 1. We proceed by induction on the number of remaining unsearched stores. The result is immediate when zero stores remains unsearched. So assume that we have shown the result for all numbers of remaining unsearched stores up to $m$. Denote a history $x_{n}=\left(\left(p_{1}, t_{1}\right),\left(p_{2}, t_{2}\right), \ldots,\left(p_{n}, t_{n}\right)\right)$ and let $p_{0}=\min \left\{p_{1}, \ldots, p_{n}\right\}$ and $t_{0}=n \tau+$ $\sum_{i=1}^{n} t_{i}$

First, suppose that $V\left(p_{0}\right)>\mathbb{E}_{t}\left[g\left(t_{0}+\tau+t\right)-g\left(t_{0}\right)\right]$. Consider the continuation strategy of continuing at $x_{n}$ and stopping at $x_{n+1}$ regardless of $\left(p_{n+1}, t_{n+1}\right)$. This strategy yields

expected utility $\int_{p_{0}}^{\infty} D(p) d p+\int_{\underline{p}}^{p_{0}} D(p) F(p) d p-\mathbb{E}_{t}\left[g\left(t_{0}+\tau+t\right)\right]>\int_{p_{0}}^{\infty} D(p) d p-g\left(t_{0}\right)$ conditional on reaching $x_{n}$ while stopping at $x_{n}$ yields utility $\int_{p_{0}}^{\infty} D(p) d p-g\left(t_{0}\right)$. By the inductive hypothesis, any strategy of the desired form yields a weakly higher expected payoff than the strategy of continuing at $x_{n}$ and stopping at $x_{n+1}$ regardless of $\left(p_{n+1}, t_{n+1}\right)$, so any such strategy yields a strictly higher expected continuation payoff conditional on reaching $x_{n}$ than does any strategy involving stopping at $x_{n}$.

Now suppose that $V\left(p_{0}\right)<\mathbb{E}_{t}\left[g\left(t_{0}+\tau+t\right)-g\left(t_{0}\right)\right]$. Consider the alternate model where search costs are fixed at $c \equiv \mathbb{E}_{t}\left[g\left(t_{0}+\tau+t\right)-g\left(t_{0}\right)\right]$. In the alternate model, it is well-known that in any sequentially rational strategy the consumer stops at $x_{n} \cdot{ }^{22}$ But, relative to expected continuation payoffs conditional on reaching $x_{n}$ in the alternate model, expected continuation payoffs conditional on reaching $x_{n}$ in the original model are the same for the strategy that stops at $x_{n}$ and are lower for any strategy that continues at $x_{n}$. So in any sequentially rational strategy in the original model the consumer stops at $x_{n}$ as well.

Therefore, any strategy of the desired form yields a strictly higher expected continuation payoff than any strategy not of this form when there are $m+1$ remaining unsearched firms, so the result for $m=N$ follows.

Proof of Proposition 7. In any minimal obfuscation NE the support of the firms' mixed strategies contains a single obfuscation level $t(p)$ for each price $p$ in the support. Every NE of the costless obfuscation model in which costly searchers enter has the distribution of prices $F(p)$ given in Proposition 3. A firm $i$ that chooses $\left(p_{i}, t_{i}\right)$ from the joint distribution of price

\footnotetext{
${ }^{22}$ See Kohn and Shavell (1974) or Weitzman (1979).
} 
and obfuscation levels in such an equilibrium cannot reduce its obfuscation level and still sell to costly searchers if and only if either $t_{i}=0$ or $\mathbb{E}_{p}\left[g\left(2 \tau+t(p)+t_{i}\right)-g\left(\tau+t_{i}\right)\right]=V\left(p_{i}\right)$. Hence, our problem is to find a function $t(p)$ such that, for every $p_{i}$,

$$
t\left(p_{i}\right)=\inf \left\{t_{i} \in \mathbb{R}^{+}: \mathbb{E}_{p}\left[g\left(2 \tau+t(p)+t_{i}\right)-g\left(\tau+t_{i}\right)\right] \geq V\left(p_{i}\right)\right\}
$$

where $p$ has cdf $F(p)$.

Let $T$ be the operator given by

$$
T(t(p))\left(p_{i}\right)=\inf \left\{t_{i} \in \mathbb{R}^{+}: \mathbb{E}_{p}\left[g\left(2 \tau+t(p)+t_{i}\right)-g\left(\tau+t_{i}\right)\right] \geq V\left(p_{i}\right)\right\}
$$

Note that $T$ is well defined by our assumptions that $\tau>0$ and $\lim _{t \rightarrow \infty} g^{\prime}(t)=\infty$. $T$ is the best-response operator when firms have lexicographic preferences for first maximizing profit and then minimizing obfuscation, given their prices, so it will suffice to show that $T$ has a fixed point and that costly searchers enter when obfuscation levels are given by a fixed point of $T$. We will proceed by applying the Schauder Fixed Point Theorem, which states that any continuous map from a nonempty, compact, and convex subset of a Banach space to itself has a fixed point.

First, note that $T$ is single-valued. Recall that the space of continuous functions $t:\left[p, p^{m}\right] \rightarrow \mathbb{R}$ under the sup norm is a Banach space. We claim that $T$ is a continuous map with respect to the topology induced by this norm. To see this, observe that if $t(p) \leq t^{\prime}(p)$ for every $p$, then $T(t(p))\left(p_{0}\right) \geq T\left(t^{\prime}(p)\right)\left(p_{0}\right)$ for every $p_{0}$. So given $t(p)$ and $\delta>0$, $\sup _{t^{\prime}(p):\left|t^{\prime}(p)-t(p)\right| \leq \delta}\left|T(t(p))-T\left(t^{\prime}(p)\right)\right|=\max \{|T(t(p))-T(t(p)+\delta)|,|T(t(p))-T(t(p)-\delta)|\}$, where $t(p)+\delta$ is notation for the function $\hat{t}(p) \equiv t(p)+\delta$ for all $p$ and $t(p)-\delta$ is defined analogously. By convexity of $g, \lim _{\delta \rightarrow 0} \max \{|T(t(p))-T(t(p)+\delta)|,|T(t(p))-T(t(p)-\delta)|\}=$ 0 , which ensures continuity of $T$.

Finally, we must show that we can restrict our attention to a nonempty, compact, and convex subset of the space of continuous functions $t:\left[p, p^{m}\right] \rightarrow \mathbb{R}$. Let $t^{0}(p)=0$ for all $p$ and let $t^{1}(p)=\inf \{t: g(2 \tau+t)-g(\tau+t) \geq V(p)\}$. Let

$$
S=\left\{t:\left[\underline{p}, p^{m}\right] \rightarrow \mathbb{R}: t \text { is continuous and } t^{0}(p) \leq t(p) \leq t^{1}(p) \text { for all } p\right\}
$$

Note that $t^{1}(p) \leq \tilde{t}$ for all $p$, so costly searchers enter if obfuscation levels are given by $t^{1}(p)$, and therefore also enter if obfuscation levels are given by $t(p)$ for any $t \in S$. Clearly, $S$ is 
nonempty, compact, and convex. Furthermore, $T$ maps $S$ to itself. For $T(t(p))\left(p_{0}\right) \geq 0$ by definition and $T(t(p))\left(p_{0}\right) \leq t^{1}\left(p_{0}\right)$ for all $t \in S$, by our observation that $T$ maps uniformly higher functions to uniformly lower ones, since $t(p) \geq t^{0}(p)$ for every $t$ in $S$. Finally, every function in the image of $T$ is continuous by our assumption that $g^{\prime \prime}$ is continuous. So $S$ is a nonempty, compact, and convex subset of a Banach space and $T: S \rightarrow S$ is continuous, so $T$ has a fixed point in $S$ by the Schauder Fixed Point Theorem, which gives a minimal obfuscation NE.

Proof of Proposition 15. The first part of the proposition is the usual condition for firms to be indifferent between charging any two prices in $\left[\underline{p}^{*}, p^{*}\right]$, as in Stahl (1989), for example.

For the second part, first note that the conditions $\int_{p^{*}}^{p^{*}} D(p) F_{p^{*}}(p) d p \geq \tau+\underline{\theta}$ and $\int_{p^{*}}^{\infty} D(p) d p \geq-\underline{\theta}$ hold if and only if there exists a $\bar{t} \geq 0$ such that $\tau+\bar{t} \leq \int_{\underline{p}^{*}}^{\infty} D(p) F_{p^{*}}(p) d p$ and $\tau+\underline{\theta}+\bar{t}=\int_{\underline{p}^{*}}^{p^{*}} D(p) F_{p^{*}}(p) d p . \quad$ For if the former two conditions hold, taking $\bar{t}=$ $\int_{\underline{p}^{*}}^{p^{*}} D(p) F_{p^{*}}(p) d p-(\tau+\underline{\theta})$ yields the latter condition, recalling that $\int_{\underline{p}^{*}}^{p^{*}} D(p) F_{p^{*}}(p) d p+$ $\int_{p^{*}}^{\infty} D(p) d p=\int_{p^{*}}^{\infty} D(p) F_{p^{*}}(p) d p$; and if the latter condition holds, $\int_{p^{*}}^{p^{*}} D(p) F_{p^{*}}(p) d p \geq \tau+\underline{\theta}$ is immediate and $\int_{p^{*}}^{\infty} D(p) d p \geq-\underline{\theta}$ follows by subtracting the equality in the latter condition from the inequality in it.

Now if there is no $\bar{t} \geq 0$ such that $\tau+\bar{t} \leq \int_{\underline{p}^{*}}^{\infty} D(p) F_{p^{*}}(p) d p$ and $\tau+\underline{\theta}+\bar{t}=$ $\int_{\underline{p}^{*}}^{p^{*}} D(p) F_{p^{*}}(p) d p$, there can be no PBE of the desired form. To see this, first note that costly searchers will not enter if the first inequality is violated and costly searchers will search for a second time if they observe price $p^{*}$ and face average obfuscation $\bar{t}$ if the righthand side of the equality is strictly greater than the left-hand side, which is impossible in PBE by Proposition 13. And if the right-hand side of the equality is strictly less than the left-hand side, then a firm would be able to profitably deviate to setting price slightly above $p>p^{*}$, as costly searchers would still buy at such a price with probability one and such a firm makes zero profit from costless searchers.

If there exists a $\bar{t} \geq 0$ such that $\tau+\bar{t} \leq \int_{\underline{p}^{*}}^{\infty} D(p) F_{p^{*}}(p) d p$ and $\tau+\underline{\theta}+\bar{t}=\int_{\underline{p}^{*}}^{p^{*}} D(p) F_{p^{*}}(p) d p$, consider the strategy profile where all firms set obfuscation level equal to $\int_{p^{*}}^{p^{*}} D(p) F_{p^{*}}(p) d p-$ $(\tau+\underline{\theta}) \geq 0$ and randomize their prices according to $F_{p^{*}}(p)$, and suppose that consumers search optimally and have the off-equilibrium path belief that $\theta=\underline{\theta}$ if they ever observe 
$p \notin\left[\underline{p}^{*}, p^{*}\right]$. Under these strategies, firms sell to all consumers who visit them first and are indifferent among all prices in $\left[\underline{p}^{*}, p^{*}\right]$, so the only deviation that could possibly be profitable would be that to a price greater that $p^{*}$. But a consumer that observes $\hat{p}>p^{*}$ expects to face search cost $\int_{p^{*}}^{p^{*}} D(p) F_{p^{*}}(p) d p-(\tau+\underline{\theta})+\tau+\underline{\theta}=\int_{p^{*}}^{p^{*}} D(p) F_{p^{*}}(p) d p$ from searching again and to receive expected benefit $\int_{\underline{p}^{*}}^{\hat{p}} D(p) F_{p^{*}}(p) d p>\int_{\underline{p}^{*}}^{p^{*}} D(p) F_{p^{*}}(p) d p$ from doing so, so a firm that deviated to such a price would not sell to consumers. So this is a PBE.

Finally, suppose that $\tau \leq \int_{\underline{p}^{m}}^{\infty} D(p) F_{p^{m}}(p) d p$. If $-\underline{\theta} \leq \int_{p^{m}}^{\infty} D(p) d p$, then a nontrivial $\mathrm{PBE}$ with $p^{*}=p^{m}$ exists by Proposition 14. So suppose that $-\underline{\theta}>\int_{p^{m}}^{\infty} D(p) d p$. Then $0<\tau+\underline{\theta}<\int_{\underline{p}^{m}}^{p^{m}} D(p) F_{p^{m}}(p) d p$. Note that $\int_{\underline{p}^{*}}^{p^{*}} D(p) F_{p^{*}}(p) d p$ equals 0 if $p^{*}$ equals 0 , equals $\int_{p^{m}}^{p^{m}} D(p) F_{p^{m}}(p) d p$ if $p^{*}$ equals $p^{m}$, and is continuous in $p^{*}$. Therefore, the Intermediate Value Theorem implies that there exists a $p^{*}$ such that $\tau+\underline{\theta}=\int_{\underline{p}^{*}}^{p^{*}} D(p) F_{p^{*}}(p) d p$. Therefore,

$$
\begin{aligned}
-\underline{\theta} & =\tau-\int_{\underline{p}^{*}}^{p^{*}} D(p) F_{p^{*}}(p) d p \\
& \leq \int_{\underline{p}^{m}}^{\infty} D(p) F_{p^{m}}(p) d p-\int_{\underline{p}^{*}}^{p^{*}} D(p) F_{p^{*}}(p) d p \\
& \leq \int_{p^{m}}^{\infty} D(p) d p \\
& \leq \int_{p^{*}}^{\infty} D(p) d p
\end{aligned}
$$

where the second inequality again uses the assumption that $\int_{\underline{p}^{*}}^{p^{*}} D(p) F_{p^{*}}(p) d p$ is increasing in $p^{*}$ for $p^{*}<p^{m}$. The characterization in the first part of the proposition then implies that a PBE exists with $\bar{p}=p^{*}$.

Proof of Proposition 16. If $p^{*}<p^{m}$, then the proof of Proposition 15 implies that the unique nontrivial PBE level of average obfuscation is $\int_{\underline{p}^{*}}^{p^{*}} D(p) F_{p^{*}}(p) d p-\underline{\theta}-\tau$, which is decreasing in $\tau$. If $p^{*}=p^{m}$, then the proof of Proposition 14 implies that nontrivial PBE average obfuscation may take on any value in between the lower bound given by $V\left(p^{m}\right)-\underline{\theta}-\tau$ and the upper bound given by $V(\infty)-\tau$, both of which are decreasing in $\tau$. So the second sentence in the proposition is proved.

Now suppose that $\tau^{\prime} \geq \tau, \bar{t}^{\prime} \geq \bar{t}$, and $\bar{t}^{\prime}$ is a nontrivial PBE average obfuscation level for 
a price distribution with supremum $\bar{p}^{\prime}$ when exogenous search costs are given by $\tau^{\prime}$, while $\bar{t}$ is a nontrivial PBE average obfuscation level for a price distribution with supremum $\bar{p}$ when exogenous search costs are given by $\tau$. We must show that $\bar{t}^{\prime}$ is also a nontrivial PBE average obfuscation level when exogenous search costs are given by $\tau$, and that $\bar{t}$ is a nontrivial PBE average obfuscation level when exogenous search costs are given by $\tau^{\prime}$.

From the proofs of Propositions 14 and 15, a nontrivial PBE with price upper bound $\bar{p}$, exogenous search costs $\tau$ and average obfuscation $\bar{t}$ satisfies $\tau+\underline{\theta}+\bar{t}=\int_{\underline{\underline{p}}}^{\bar{p}} D(p) F_{\bar{p}}(p) d p$ if $\bar{p}<p^{m}$ and $\tau+\underline{\theta}+\bar{t} \geq \int_{\underline{p}}^{\bar{p}} D(p) F_{\bar{p}}(p) d p$ if $\bar{p}=p^{m}$. By Assumption 1, this implies that $\bar{p}^{\prime} \geq \bar{p}$, since $\tau^{\prime} \geq \tau$ and $\bar{t}^{\prime} \geq \bar{t}$.

If $\tau+\underline{\theta}+\bar{t}^{\prime}>\int_{\underline{\underline{p}}^{m}}^{p^{m}} D(p) F_{p^{m}}(p) d p$, then by the assumption that $\tau^{\prime} \geq \tau$ and Assumption $1, \tau^{\prime}+\underline{\theta}+\bar{t}^{\prime}>\int_{\bar{p}}^{\bar{p}} D(p) F_{\bar{p}}(p) d p$ for all $\bar{p}<p^{m}$, so we must have $\bar{p}^{\prime}=p^{m}$. This implies that $\tau^{\prime}+\bar{t}^{\prime} \leq \int_{\underline{p}^{m}}^{\infty} D(p) F_{p^{m}}(p) d p$, so $\tau+\bar{t}^{\prime} \leq \int_{\underline{p}^{m}}^{\infty} D(p) F_{p^{m}}(p) d p$, in which case $\bar{t}^{\prime}$ is a nontrivial PBE average obfuscation level for a price distribution with supremum $p^{m}$ when exogenous search costs are given by $\tau$, by Proposition 14. Otherwise, we have $\int_{\underline{\bar{p}}}^{\bar{p}} D(p) F_{\bar{p}}(p) d p \leq \tau+$ $\underline{\theta}+\bar{t}^{\prime} \leq \int_{\underline{\bar{p}}^{\prime}}^{\bar{p}^{\prime}} D(p) F_{\bar{p}^{\prime}}(p) d p$. Since $\int_{\underline{p}^{*}}^{p^{*}} D(p) F_{p^{*}}(p) d p$ is continuous in $p^{*}$, by the Intermediate Value Theorem there exits $p^{*}$ such that $\tau+\underline{\theta}+\bar{t}^{\prime}=\int_{\underline{p}^{*}}^{p^{*}} D(p) F_{p^{*}}(p) d p$, which combined with the facts that $\int_{\underline{\underline{p}}}^{\infty} D(p) d p \geq-\underline{\theta}, \int_{\underline{\bar{p}}^{\prime}}^{\infty} D(p) d p \geq-\underline{\theta}, \bar{p} \leq p^{*} \leq \bar{p}^{\prime}$, and Proposition 15 implies that $\bar{t}^{\prime}$ is a nontrivial PBE average obfuscation level for a price distribution with supremum $p^{*}$ when exogenous search costs are given by $\tau$.

The argument for why $\bar{t}$ is a nontrivial PBE average obfuscation level when exogenous search costs are given by $\tau^{\prime}$ is very similar.

Proof of Proposition 17. Let $u, u^{\prime}, \tau$, and $\tau^{\prime}$ be as in the proof of Proposition 5. Suppose throughout that $u$ is the value of consumer welfare for a nontrivial PBE with price upper bound $\bar{p}$ and that $u^{\prime}$ is the value of consumer welfare for a nontrivial PBE with price upper bound $\bar{p}^{\prime}$. Let $\bar{t}\left(\bar{t}^{\prime}\right)$ be the average obfuscation level in a nontrivial PBE with price upper bound $\bar{p}\left(\bar{p}^{\prime}\right)$ that yields costly searcher welfare $u\left(u^{\prime}\right)$ when exogenous search costs are $\tau$ $\left(\tau^{\prime}\right)$.

We first claim that $\bar{p} \geq \bar{p}^{\prime}$. This is trivial if $\bar{p}=p^{m}$, so suppose that $\bar{p}<p^{m}$. By the proof of Proposition 15, $u=\int_{\bar{p}}^{\infty} D(p) d p+\underline{\theta}$, and, by the proofs of Propositions 14 and 15, $u^{\prime} \leq \int_{\bar{p}^{\prime}}^{\infty} D(p) d p+\underline{\theta}$. So since $u^{\prime} \geq u$ it must be the case that $\bar{p}^{\prime} \leq \bar{p}$. 
Next, we note that the set of nontrivial PBE prices is increasing in $\tau$ in the strong set order. Restricting attention to price distributions with $\bar{p}<p^{m}$, this follows immediately from Proposition 15 and Assumption 1. To see that there is no problem caused by $\mathrm{PBE}$ with $\bar{p}=p^{m}$, recall from Proposition 14 that such a PBE exists if and only if $\tau \leq V(\infty)$ and $\int_{p^{m}}^{\infty} D(p) d p \geq-\underline{\theta}$. Therefore, the set of nontrivial PBE prices must be increasing in $\tau$ in the strong set order if there cannot be nontrivial $\mathrm{PBE}$ when $\tau>V(\infty)$ and $\int_{p^{m}}^{\infty} D(p) d p \geq-\underline{\theta}$. This is true because $\tau+\underline{\theta} \leq \int_{\bar{p}}^{\bar{p}} D(p) F_{\bar{p}}(p) d p$ and $\bar{p}<p^{m}$ imply that $\tau+\underline{\theta}<\int_{\underline{p}^{m}}^{p^{m}} D(p) F_{p^{m}}(p) d p$, by Assumption 1; so if $\tau>V(\infty)$, then $-\underline{\theta}>\int_{p^{m}}^{\infty} D(p) d p$.

We consider separately the cases where $\bar{p}<p^{m}$ and where $\bar{p}=p^{m}$. If $\bar{p}<p^{m}$, the fact that the set of nontrivial PBE prices is increasing in $\tau$ in the strong set order implies that a nontrivial PBE with price upper bound equal to $\bar{p}$ exists when exogenous search costs equal $\tau^{\prime}$. Recall from the proof of Proposition 15 that in a nontrivial PBE with price upper bound $\bar{p}<p^{m}$, exogenous search costs $\tau$ and average obfuscation $\bar{t}$, costly searcher welfare equals $\int_{\bar{p}}^{\infty} D(p) d p+\underline{\theta}$, since $\tau+\bar{t}=\int_{\underline{\underline{p}}}^{\bar{p}} D(p) F_{\bar{p}}(p) d p-\underline{\theta}$. This does not depend on $\tau$ or $\bar{t}$, so a nontrivial PBE with price upper bound equal to $\bar{p}$ and exogenous search costs $\tau^{\prime}$ yields costly searcher welfare $u$. Similarly, if $\bar{p}<p^{m}$ then $\bar{p}^{\prime}<p^{m}$ and there exists a nontrivial PBE with price upper bound equal to $\bar{p}^{\prime}$ when exogenous search costs equal $\tau$, and such a PBE yields costly searcher welfare $u^{\prime}$

Finally, suppose that $\bar{p}=p^{m}$. By Proposition 14, this implies that $\int_{p^{m}}^{\infty} D(p) d p \geq-\underline{\theta}$. Since the set of nontrivial PBE prices is increasing in $\tau$ in the strong set order and is nonempty when exogenous search costs equal $\tau^{\prime}$, a nontrivial PBE with price upper bound $p^{m}$ exists when exogenous search costs equal $\tau^{\prime}$, so $\tau^{\prime} \leq V(\infty)$. Letting $\bar{t}^{*}$ take values between $\int_{\underline{p}^{m}}^{p^{m}} D(p) F_{p^{m}}(p) d p$ and $V(\infty)-\tau^{\prime}$, we see that nontrivial PBE with price upper bound $p^{m}$ exist that yield every value of costly searcher welfare between $\int_{p^{m}}^{\infty} D(p) d p+\underline{\theta}$ and 0 , when exogenous search costs equal $\tau^{\prime}$. So if $u \leq \int_{p^{m}}^{\infty} D(p) d p+\underline{\theta}$, we already have shown that a nontrivial PBE yielding costly searcher welfare $u$ exists when exogenous search costs equal $\tau^{\prime}$. If $u>\int_{p^{m}}^{\infty} D(p) d p+\underline{\theta}$, recall that $u \leq u^{\prime}$, the costly searcher welfare when price upper bound equals $\bar{p}^{\prime}$ and exogenous search costs equal $\tau^{\prime}$. This implies that $\bar{p}^{\prime}<p^{m}$, and $u^{\prime}=\int_{\bar{p}^{\prime}}^{\infty} D(p) d p+\underline{\theta}$. Since $\int_{\bar{p}}^{\infty} D(p) d p$ is increasing in $\bar{p}$ for $\bar{p}<p^{m}$, by the Intermediate Value Theorem there exists $\bar{p}^{*} \in\left(\bar{p}^{\prime}, p^{m}\right)$ such that $\int_{\bar{p}^{*}}^{\infty} D(p) d p+\underline{\theta}=u$. By Proposition 15 , 
there exists a nontrivial PBE when exogenous search costs equal $\tau^{\prime}$ with price upper bound $\bar{p}^{*}$ that yields costly searcher welfare $u$.

It remains only to show that if $\bar{p}=p^{m}$, there exists a nontrivial PBE when exogenous search costs equal $\tau$ that yields costly consumer surplus $u^{\prime}$. If $\bar{p}^{\prime}<p^{m}$, then this follows exactly as in the case where $\bar{p}<p^{m}$. If $\bar{p}^{\prime}=p^{m}$, then it can easily be checked that there exists a nontrivial PBE with exogenous search costs $\tau$, price upper bound $p^{m}$, and average obfuscation $\bar{t}^{*} \equiv \bar{t}^{\prime}+\tau^{\prime}-\tau$ yielding costly searcher surplus $u^{\prime}$. 


\section{References}

Baye, Michael and John Morgan (2001), "Information Gatekeepers on the Internet and the Competitiveness of Homogeneous Product Markets," American Economic Review, 91, 454-474.

Baye, Michael, John Morgan, and Patrick Scholten (2006), "Information, Search, and Price Dispersion," in T. Hendershott (ed.) Handbook of Economics and Information Systems, Elsevier Press, Amsterdam.

Burdett, Kenneth and Kenneth L. Judd (1983), "Equilibrium Price Dispersion," Econometrica, 51, 955-969.

Butters, Gerard (1977), "Equilibrium Distributions of Sales and Advertising Prices," Review of Economic Studies, 44, 465-491.

Carlin, Bruce Ian (2008), "Strategic Price Complexity in Retail Financial Markets," Journal of Financial Economics, forthcoming.

Diamond, Peter (1971), "A Model of Price Adjustment," Journal of Economic Theory, 3, 156-168.

Eliaz, Kfir and Ran Spiegler (2008), "Consideration Sets and Competitive Marketing," mimeo, Brown University and London School of Economics.

Ellison, Glenn (2005), “A Model of Add-on Pricing," Quarterly Journal of Economics, 120, 585-637.

Ellison, Glenn (2006), "Bounded Rationality in Industrial Organization," in Richard Blundell, Whitney Newey, and Torsten Persson (eds.) Advances in Economics and Econometrics: Theory and Applications, Ninth World Congress, Cambridge University Press, Cambridge.

Ellison, Glenn and Sara Fisher Ellison (2009), "Search, Obfuscation, and Price Elasticities on the Internet," Econometrica, 77, 427-452.

Gabaix, Xavier and David Laibson (2006), "Shrouded Attributes, Consumer Myopia, and Information Suppression in Competitive Markets," Quarterly Journal of Economics, 121, 505-540.

Grossman, Sanford J. (1981), "The Role of Warranties and Private Disclosure about Product Quality," Journal of Law and Economics, 24, 461-483.

Kohn, Meir and Steven Shavell (1974), "The Theory of Search," Journal of Economic Theory, 9, 93-123.

Milgrom, Paul (1981), "Good News and Bad News: Representation Theorems and Applications," Bell Journal of Economics, 12, 380-391.

Rosenthal, Robert W. (1980), "A Model in which an Increase in the Number of Sellers 
Leads to a Higher Price," Econometrica, 48, 1575-1579.

Salop, Stephen and Joseph Stiglitz (1977), "Bargains and Ripoffs: A Model of Monopolistically Competitive Price Dispersion," Review of Economics Studies, 49, 3-13.

Spiegler, Ran (2006), "Competition over Agents with Boundedly Rational Expectations," Theoretical Economics, 1, 207-231.

Stahl, Dale O. (1989), "Oligopolistic Pricing with Sequential Consumer Search." American Economic Review, 79, 700-712.

Stahl, Dale O. (1996), "Oligopolistic Pricing with Heterogeneous Consumers Search," International Journal of Industrial Organization, 14, 243-268.

Varian, Hal (1980), "A Model of Sales," American Economic Review, 70, 651-659.

Weitzman, Martin (1979), "Optimal Search for the Best Alternative," Econometrica, 47, 641-654.

Wilson, Chris M. (2008), "Ordered Search and Equilibrium Obfuscation," University of Oxford, mimeo. 